Pure and Applied Mathematics Quarterly

Volume 6, Number 2

(Special Issue: In honor of

Michael Atiyah and Isadore Singer)

555 - 601, 2010

\title{
K-Homology, Assembly and Rigidity Theorems for Relative Eta Invariants
}

\author{
Nigel Higson and John Roe
}

\begin{abstract}
We connect the assembly map in $\mathrm{C}^{*}$-algebra $\mathrm{K}$-theory to rigidity properties for relative eta invariants that have been investigated by Mathai, Keswani, Weinberger and others. We give a new and conceptual proof of Keswani's theorem that whenever the $C^{*}$-algebra assembly map is an isomorphism, the relative eta invariants associated to the signature operator are homotopy invariants, whereas the relative eta invariants associated to the Dirac operator on a manifold with positive scalar curvature vanish.
\end{abstract}

Keywords: K-homology, relative eta-invariant, $\mathbb{R} / \mathbb{Z}$-index, Baum-Connes conjecture.

\section{INTRODUCTION}

The purpose of this paper is to examine connections between K-homology theory and relative eta invariants. Our aim is to place certain rigidity theorems for relative eta invariants into the context of Baum's geometric K-homology theory [8]. We hope to persuade the reader that this is a natural environment for them.

The eta invariant of a self-adjoint linear elliptic partial differential operator $\mathrm{D}$ on a closed manifold $M$ is a regularization, introduced by Atiyah, Patodi and Singer $[2,3]$, of the difference between the dimensions of the positive and negative eigenspaces of D. It depends sensitively on the operator. A more robust version

Received April 13, 2008.

The authors were partially supported by a US National Science Foundation grant. 
is the relative eta invariant, associated to $D$ together with a map $f: M \rightarrow B \pi$ for some discrete group $\pi$, and a pair of group homomorphisms $\sigma_{1}, \sigma_{2}: \pi \rightarrow U(N)$. This is, roughly speaking, half the difference of the eta invariants of the two elliptic operators that are obtained by "twisting" $D$ by the pull-backs, via the map $f$, of the flat bundles on $B \pi$ associated to $\sigma_{1}$ and $\sigma_{2}$ :

$$
\rho\left(D, f, \sigma_{1}, \sigma_{2}\right)=\frac{1}{2} \eta\left(D_{1}\right)-\frac{1}{2} \eta\left(D_{2}\right)
$$

(the actual difference involves a correction term; this and the reason for the factor of $\frac{1}{2}$ will be reviewed in Section 2).

We shall present an analysis of relative eta invariants that is based on the $\mathrm{C}^{*}$-algebraic assembly map

$$
\mu: \mathrm{K}_{*}(\mathrm{~B} \pi) \longrightarrow \mathrm{K}_{*}\left(\mathrm{C}^{*}(\pi)\right) .
$$

The groups $K_{*}(B \pi)$ appearing here are those of the generalized homology theory that is dual to Atiyah-Hirzebruch K-theory. They may be concretely defined using either functional analysis [25, 12] (as first suggested by Atiyah [1]) or geometry $[8,10]$. We shall use both realizations in this paper. The target groups for the assembly map are the $\mathrm{K}$-theory groups of the group $\mathrm{C}^{*}$-algebra for $\pi$. Thus $\mathrm{K}_{0}\left(C^{*}(\pi)\right)$ is the usual algebraic $\mathrm{K}$-theory group, while $\mathrm{K}_{1}\left(\mathrm{C}^{*}(\pi)\right)$ is the component group of the stable general linear group of $C^{*}(\pi)\left(C^{*}\right.$-algebra $\mathrm{K}$-theory groups are Bott-periodic, so there are no further groups to define). The assembly map, as we shall study it, here was introduced by Kasparov [25] and is central to the $\mathrm{C}^{*}$-algebra approach to the Novikov conjecture.

Our interest is in the following results, originally due in various formulations to Mathai [29], Weinberger [37] and Keswani [27] (for an interesting recent treatment, see the article [32] of Piazza and Schick).

1.1. Theorem. Let D be the Dirac operator on a closed, odd-dimensional spinmanifold $\mathrm{M}$ with positive scalar curvature. If the assembly map

$$
\mu: \mathrm{K}_{*}(\mathrm{~B} \pi) \longrightarrow \mathrm{K}_{*}\left(\mathrm{C}^{*}(\pi)\right)
$$

is an isomorphism, then for any map $\mathrm{f}: \mathrm{M} \rightarrow \mathrm{B} \pi$ and any pair of representations $\sigma_{1}, \sigma_{2}: \pi \rightarrow \mathrm{U}(\mathrm{N})$ the relative eta invariant $\rho\left(\mathrm{D}, \mathrm{f}, \sigma_{1}, \sigma_{2}\right)$ is equal to zero.

1.2. Theorem. Let $\mathrm{h}: \mathrm{M}^{\prime \prime} \rightarrow \mathrm{M}^{\prime}$ be an orientation-preserving homotopy equivalence between odd-dimensional, closed, oriented manifolds. Let $\mathrm{f}^{\prime}: \mathrm{M}^{\prime} \rightarrow \mathrm{B} \pi$ 
be any map, and let $\mathrm{f}^{\prime \prime}=\mathrm{f}^{\prime} \circ \mathrm{h}: \mathrm{M}^{\prime \prime} \rightarrow \mathrm{B} \pi$. Let $\mathrm{D}^{\prime}$ and $\mathrm{D}^{\prime \prime}$ be the signature operators on $\mathrm{M}^{\prime}$ and $\mathrm{M}^{\prime \prime}$. If the assembly map

$$
\mu: \mathrm{K}_{*}(\mathrm{~B} \pi) \longrightarrow \mathrm{K}_{*}\left(\mathrm{C}^{*}(\pi)\right)
$$

is an isomorphism, then for any pair of representations $\sigma_{1}, \sigma_{2}: \pi \rightarrow \mathrm{U}(\mathrm{N})$, the relative eta invariants

$$
\rho\left(D^{\prime}, f^{\prime}, \sigma_{1}, \sigma_{2}\right) \quad \text { and } \rho\left(D^{\prime \prime}, f^{\prime \prime}, \sigma_{1}, \sigma_{2}\right)
$$

are equal to one another.

There is a more refined assembly map in $\mathrm{C}^{*}$-algebra K-theory, introduced by Baum and Connes [6, 7]. It treats torsion in $\pi$ carefully and also pays due attention to the different ways in which the group algebra of $\pi$ may be completed into a $C^{*}$-algebra. Baum and Connes conjecture that their assembly map is an isomorphism for all groups $\pi$. If, for example, $\pi$ is a torsion-free and amenable group, then the Baum-Connes assembly map agrees with the one studied in this paper. The same holds more generally for torsion-free a-T-menable groups [13], and for these the Baum-Connes conjecture was proved in [20].

To prove Theorems 1.1 and 1.2 we shall follow an approach that was pioneered in the thesis of Keswani [27]. Indeed the main contribution of our paper is to conceptualize Keswani's work. Here is a brief account of our method, focused mostly on Theorem 1.1.

In an earlier sequence of papers $[22,23,24]$ we introduced and analyzed "analytic structure groups" $\mathcal{S}_{*}(\pi)$ that measure the failure of the assembly map to be an isomorphism. They fit into a long exact sequence

$$
\begin{aligned}
\cdots \longrightarrow \mathrm{K}_{0}(\mathrm{~B} \pi) \stackrel{\mu}{\longrightarrow} \mathrm{K}_{0}\left(\mathrm{C}^{*}(\pi)\right) & \longrightarrow \mathcal{S}_{1}(\pi) \\
& \longrightarrow \mathrm{K}_{1}(\mathrm{~B} \pi) \stackrel{\mu}{\longrightarrow} \mathrm{K}_{1}\left(\mathrm{C}^{*}(\pi)\right) \longrightarrow \cdots,
\end{aligned}
$$

and are so named because they are $\mathrm{C}^{*}$-algebra K-theory counterparts of the structure groups in surgery theory $[36,33]$.

Atiyah showed in [1] that each elliptic operator D on a closed manifold $M$ determines a class $[D] \in K_{*}(M)$. The assembly map sends this class to an equivariant analytic index obtained by causing $\mathrm{D}$ to act on sections of a canonical flat line bundle over $M$ whose fibers are $C^{*}(\pi)$-modules $[30,25]$. 
Now let $\mathrm{D}$ be the Dirac operator on an odd-dimensional spin-manifold $M$ that is equipped with a positive scalar curvature metric. If $f: M \rightarrow B \pi$ is any map, then it follows from the Lichnerowicz formula that the class $f_{*}[D] \in K_{1}(B \pi)$ maps to zero under the assembly map. Therefore $f_{*}[D]$ pulls back to a class in the analytic structure group $\mathcal{S}_{1}(\pi)$. We shall use the positive scalar curvature of $M$ to identify a particular such pullback that we shall denote by $[M, f] \in \mathcal{S}_{1}(\pi)$.

Here is the connection with relative eta invariants. Each representation $\sigma: \pi \rightarrow$ $\mathrm{U}(\mathrm{N})$ determines a trace functional

$$
\operatorname{Tr}_{\sigma}: K_{0}\left(C^{*}(\pi)\right) \longrightarrow \mathbb{Z}
$$

Its composition with the assembly map $\mu: \mathrm{K}_{0}(\mathrm{~B} \pi) \rightarrow \mathrm{K}_{0}\left(\mathrm{C}^{*}(\pi)\right)$ depends only on the rank $N$ of the representation. This implies that if $\sigma_{1}$ and $\sigma_{2}$ have the same rank, then the difference

$$
\operatorname{Tr}_{\sigma_{1}}-\operatorname{Tr}_{\sigma_{2}}: K_{0}\left(C^{*}(\pi)\right) \longrightarrow \mathbb{Z}
$$

factors through the structure group $\mathcal{S}_{1}(\pi)$ in the analytic surgery long exact sequence. We shall explicitly construct a commuting diagram

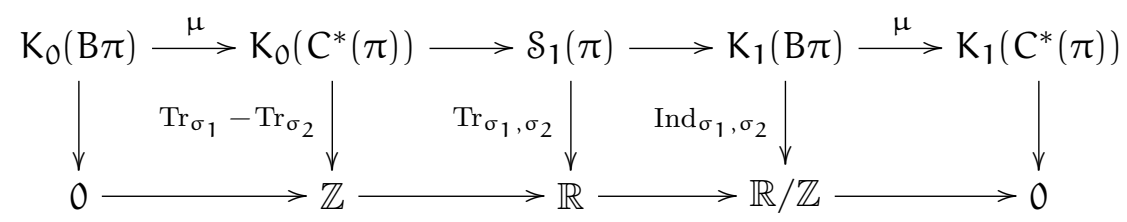

that expresses this fact and also incorporates the $\mathbb{R} / \mathbb{Z}$-index of Atiyah, Patodi and Singer [5].

We shall give a geometric account of the relative trace map

$$
\operatorname{Tr}_{\sigma_{1}, \sigma_{2}}: \mathcal{S}_{1}(\pi) \rightarrow \mathbb{R}
$$

starting from the geometric approach to K-homology in which cycles are given by elliptic operators on manifolds. As we shall review in Section 3, the equivalence relation among cycles for K-homology involves several operations (for example, bordism) that were devised with the Atiyah-Singer index theorem in mind. Thus all the operations preserve the analytic index of elliptic operators. A key observation, made in Section 6 , is that the same operations also preserve the relative eta invariant, modulo $\mathbb{Z}$. This allows us to give a concise account of the $\mathbb{R} / \mathbb{Z}$-index. 
To obtain a relative trace with values in $\mathbb{R}$ rather than $\mathbb{R} / \mathbb{Z}$ we need to equip the elliptic operators that determine K-homology cycles with "cuts" in their spectrum, separating $\pm \infty$. As it turns out, the notion of spectral cut is precisely what is needed to pull back classes in $K_{1}(B \pi)$ to the analytic structure group $\mathcal{S}_{1}(\pi)$ (compare [15]). It therefore seems to us that the fit between the analytic surgery sequence of [24] and relative eta invariants is extremely close.

There is another, more analytic approach to the relative trace map, in which it is defined as regularized difference of actual traces (this was our original approach; hence the name). But this we shall explain elsewhere.

Returning now to the proofs of Theorems 1.1 and 1.2, if $\mathrm{D}$ is the Dirac operator on an odd-dimensional spin-manifold with positive scalar curvature, and if $[M, f]$ is the class in $S_{1}(\pi)$ that is associated to the given positive scalar curvature metric on $M$, then we shall see that, more or less by definition,

$$
\operatorname{Tr}_{\sigma_{1}, \sigma_{2}}([M, f])=\rho\left(D, f, \sigma_{1}, \sigma_{2}\right) .
$$

If the assembly map is an isomorphism, then of course the structure group $\mathcal{S}_{1}(\pi)$ vanishes, and in particular the class $[M, f]$ is zero. We therefore find that in this case

$$
\rho\left(D, f, \sigma_{1}, \sigma_{2}\right)=\operatorname{Tr}_{\sigma_{1}, \sigma_{2}}([M, f])=0,
$$

and we have proved Theorem 1.1.

The case of the signature operator is similar. Given an orientation-preserving homotopy equivalence $h: M^{\prime \prime} \rightarrow M^{\prime}$ between closed, oriented, odd-dimensional manifolds, the difference

$$
f_{*}^{\prime}\left[D^{\prime}\right]-f_{*}^{\prime \prime}\left[D^{\prime \prime}\right] \in K_{1}(B \pi)
$$

of the signature operator classes for $M^{\prime}$ and $M^{\prime \prime}$ maps to zero in $K_{1}\left(C^{*}(\pi)\right)$ under the assembly map. There is a class $\left[h, f^{\prime}, f^{\prime \prime}\right] \in S_{1}(\pi)$ that accounts for this, and moreover

$$
\operatorname{Tr}_{\sigma_{1}, \sigma_{2}}\left(\left[h, f^{\prime}, f^{\prime \prime}\right]\right)=\rho\left(D^{\prime}, f^{\prime}, \sigma_{1}, \sigma_{2}\right)-\rho\left(D^{\prime \prime}, f^{\prime \prime}, \sigma_{1}, \sigma_{2}\right)
$$

Theorem 1.2 follows. 


\section{Relative Eta Invariants}

We shall quickly review those aspects of the theory of eta invariants that we shall need. For further information we refer the reader to the original articles of Atiyah, Patodi and Singer [3, 4, 5], or to the monograph of Gilkey [17].

Let $\mathrm{D}$ be a formally self-adjoint, first-order elliptic partial differential operator on a closed Riemannian manifold $M$, acting on smooth sections of a Hermitian bundle $S$ over $M$ (in all our examples, $M$ will be odd-dimensional). Viewed as an unbounded symmetric operator on the Hilbert space $L^{2}(M, S)$ with domain the smooth sections of $S$, the operator $D$ is essentially self-adjoint and its closure has compact resolvent. There is therefore an orthonomal basis $\left\{\phi_{n}\right\}$ for $L^{2}(M, S)$ consisting of eigenvectors for D:

$$
\mathrm{D} \phi_{\mathrm{n}}=\lambda_{\mathrm{n}} \phi_{\mathrm{n}}
$$

(D acts initially in the sense of distributions, although the $\phi_{n}$ turn out to be smooth sections of $\mathrm{S}$ ). The eta function for $\mathrm{D}$ is the complex function

$$
\eta_{D}(s)=\sum_{n} \operatorname{sign}\left(\lambda_{n}\right)\left|\lambda_{n}\right|^{-s}
$$

where we define $\operatorname{sign}(0)$ to be 0 . The series converges absolutely as long as $\operatorname{Re}(s) \gg 0$, and moreover $\eta_{\mathrm{D}}(\mathrm{s})$ admits a meromorphic continuation to $\mathbb{C}$. An important theorem of Atiyah, Patodi and Singer asserts that the eta function is regular at $s=0$. The eta invariant of $\mathrm{D}$ is by definition the value of the eta function at this place:

$$
\eta(D)=\eta_{D}(0)
$$

Atiyah, Patodi and Singer connected the eta invariant with index theory, as follows.

2.1. Definition. Suppose that $W$ is a compact Riemannian manifold with boundary $M$, and suppose that $W$ is isometric to the Riemannian product $[0,1) \times M$ in a collaring neighborhood of $M$. Suppose that there is a $\mathbb{Z} / 2 \mathbb{Z}$-graded, formally self-adjoint, first-order elliptic partial differential operator $\mathrm{Q}$ on $\mathrm{W}$, acting on the smooth sections of a $\mathbb{Z} / 2 \mathbb{Z}$-graded Hermitian bundle over $W$ that is isomorphic to $S \oplus S$ on the collaring neighborhood. Suppose finally that in the collaring 
neighborhood Q has the form

$$
Q=\left(\begin{array}{cc}
0 & -\partial+D \\
\partial+D & 0
\end{array}\right)
$$

where $\partial$ denotes differentiation on $(0,1]$. Under all these circumstances we shall say that $\mathrm{Q}$ is bounded by $\mathrm{D}$ and that $\mathrm{D}$ is a boundary.

Now let $P: L^{2}(M, S) \rightarrow L^{2}(M, S)$ be the projection onto direct sum of the nonnegative eigenspaces of the operator D. Atiyah, Patodi and Singer showed that if $\mathrm{Q}$, as in Definition 2.1, is restricted so as to act only on smooth sections $\left(\mathrm{s}^{+}, \mathrm{s}^{-}\right)$ that satisfy the boundary conditions

$$
\left.\mathrm{Ps}^{+}\right|_{\mathrm{M}}=0 \text { and }\left.\quad \mathrm{P}^{\perp} \mathrm{s}^{-}\right|_{\mathrm{M}}=0
$$

then it becomes a Fredholm operator. We shall denote by $\operatorname{Ind}(\mathrm{Q}, \mathrm{D})$ the index of the part of $\mathrm{Q}$ that maps even sections satisfying the boundary conditions to arbitrary odd sections; this is the Atiyah-Patodi-Singer index of Q.

Let $Z$ be an extension of $W$ to a closed Riemannian manifold and assume that the operator $\mathrm{Q}$ extends to a $\mathbb{Z} / 2 \mathbb{Z}$-graded, formally self-adjoint, first-order elliptic partial differential operator on $Z$ (for example, $Z$ could be the double of $W)$. Let $\varepsilon$ be the grading operator. As is well known, the operators $\exp \left(-t Q^{2}\right)$ are trace-class for $t>0$ and there is an asymptotic expansion

$$
\operatorname{Trace}\left(\varepsilon \exp \left(-\mathrm{t} \mathrm{Q}^{2}\right)\right) \sim \sum_{k \geq-\frac{n}{2}} a_{k} t^{k} .
$$

Moreover each coefficient $a_{k}$ is the integral over $Z$ of a smooth function $a_{k}(z)$ that is locally computable from the coefficients of $Q$ and their derivatives.

The fundamental Atiyah, Patodi Singer (APS) index theorem is as follows:

2.2. Theorem. ([3, Theorem 3.10].) If the operator $\mathrm{Q}$ on $\mathrm{W}$ is bounded by $\mathrm{D}$, then

$$
\operatorname{Ind}(Q, D)=\int_{W} a_{0}(w) d w-\frac{1}{2}(\eta(D)-\operatorname{dim} \operatorname{ker}(D))
$$

Now let $\pi$ be a discrete group and let B $\pi$ be a classifying space for $\pi$. Suppose that the manifold $M$ is equipped with a continuous map

$$
\mathrm{f}: \mathrm{M} \rightarrow \mathrm{B} \pi \text {. }
$$


The map determines a principal $\pi$-space $\tilde{M}$ over $M$, and every group homomorphism $\sigma: \pi \rightarrow \mathrm{U}(\mathrm{N})$ therefore determines a flat Hermitian vector bundle

$$
\mathrm{V}_{\sigma}=\tilde{M} \times_{\pi} \mathbb{C}^{\mathrm{N}}
$$

Since $V_{\sigma}$ is flat, we can lift $D$ to an operator $D_{\sigma}$ acting on sections of $V_{\sigma} \otimes S$ and then form the eta invariant $\eta\left(D_{\sigma}\right)$.

2.3. Definition. Let $\sigma_{1}$ and $\sigma_{2}$ be two homomorphisms from $\pi$ into the unitary group $\mathrm{U}(\mathrm{N})$. The relative eta invariant $\rho\left(\mathrm{D}, \mathrm{f}, \sigma_{1}, \sigma_{2}\right)$ associated to a formally self-adjoint, first-order elliptic partial differential operator on a closed Riemannian manifold $M$ and a map $f: M \rightarrow B \pi$ is the quantity

$$
\rho\left(D, f, \sigma_{1}, \sigma_{2}\right)=\frac{1}{2}\left(\eta\left(D_{1}\right)-\eta\left(D_{2}\right)\right)-\frac{1}{2}\left(\operatorname{dim} \operatorname{ker}\left(D_{1}\right)-\operatorname{dim} \operatorname{ker}\left(D_{2}\right)\right)
$$

(we have written $D_{1}$ and $D_{2}$ in place of $D_{\sigma_{1}}$ and $D_{\sigma_{2}}$, and we shall continue to do so from now on).

Relative eta invariants behave in many ways more simply than the individual eta invariants out of which they are constructed. For example, it is quite easy to see that the difference of the eta functions that define $\eta\left(D_{1}\right)$ and $\eta\left(D_{2}\right)$ is not only meromorphic on $\mathbb{C}$ but in fact entire. Indeed we can write

$$
\begin{aligned}
\eta_{D_{1}}(s)-\eta_{D_{2}}(s) & = \\
& \Gamma\left(\frac{s+1}{2}\right)^{-1} \int_{0}^{\infty}\left(\operatorname{Trace}\left(D_{1} e^{-t D_{1}^{2}}\right)-\operatorname{Trace}\left(D_{2} e^{-t D_{2}^{2}}\right)\right) t^{\frac{s-1}{2}} d t
\end{aligned}
$$

and basic facts about heat kernel asymptotics imply that this integral is absolutely convergent for all $s$.

Key for us will be the following simple consequence of the Atiyah-Patodi-Singer index theorem (compare [4, Section 3]):

2.4. Lemma. Assume that $\mathrm{D}$ is bounded by some operator $\mathrm{Q}$, as above, and assume that the map $\mathrm{f}: \mathrm{M} \rightarrow \mathrm{B} \pi$ extends to the compact manifold $\mathrm{W}$. Under these circumstances, the relative eta invariant $\rho\left(D, f, \sigma_{1}, \sigma_{2}\right)$ is an integer.

Proof. The $\mathrm{a}_{0}$-integrals for $\mathrm{D}_{1}$ and $\mathrm{D}_{2}$ in Theorem 2.2 are equal because the integrands are locally determined from $\mathrm{D}_{1}$ and $\mathrm{D}_{2}$, and locally these operators are isomorphic to one another. It therefore follows from Theorem 2.2 that

$$
\operatorname{Ind}\left(Q_{1}, D_{1}\right)-\operatorname{Ind}\left(Q_{2}, D_{2}\right)=-\rho\left(D, f, \sigma_{1}, \sigma_{2}\right),
$$


which proves integrality.

2.5. Remark. This integrality result is the reason that the factor of one half, and also the correction term involving the kernel, are included in the definition of the relative eta invariant.

\section{Geometric K-Homology}

In this section we shall review Baum's geometric definition of K-homology, essentially as presented in his article [8] with Douglas (see also [10]). We shall actually use the small variant of Baum's original definition that is presented by Keswani in [26]. It better suits our treatment of the signature operator and more transparently explains the connection to relative eta invariants. We shall focus on the odd-degree K-homology group, since this is the one most relevant to relative eta invariants.

The geometric definition of K-homology involves cycles and an equivalence relation between cycles. The cycles are based on the following notion:

3.1. Definition. Let $M$ be an Riemannian manifold. A Dirac bundle for $M$ is a smooth, complex Hermitian vector bundle $S$ on $M$ that is equipped with a smooth, $\mathbb{R}$-linear bundle map

$$
c: \mathrm{TM} \longrightarrow \operatorname{End}(\mathrm{S})
$$

such that $c(Y)^{*}=-c(Y)$ and $c(Y)^{2}=-\|Y\|^{2}$ for all tangent vectors $Y$. If $M$ is even-dimensional, then in addition we require that $S$ be $\mathbb{Z} / 2 \mathbb{Z}$-graded, and that the action $\mathrm{c}: \mathrm{TM} \rightarrow \operatorname{End}(\mathrm{S})$ exchange the even and odd-graded components of $S$.

We have used the term Dirac bundle to be consistent with the monograph [21]; the term Clifford bundle is used elsewhere. We shall refer to the map c as the Clifford action of TM on S.

3.2. Definition. Let $\mathrm{X}$ be any space. An (odd) geometric $\mathrm{K}$-cycle for $\mathrm{X}$ is a triple $(M, S, f)$ where:

(a) $M$ is a closed, orientable Riemannian manifold whose connected components all have odd dimension (they need not have the same dimension).

(b) $S$ is a Dirac bundle on $M$.

(c) $f$ is a continuous map from $M$ to $X$. 
3.3. Remark. The definition in [26] adds to the concept of cycle a suitable connection on $\mathrm{S}$. This is an inessential difference that does not alter the geometric K-homology groups we are about to define.

3.4. Definition. Let $(M, S, f)$ be a geometric K-cycle for $X$. It is a boundary if there exist

(a) a compact Riemannian manifold $W$ with boundary $M$ such that $W$ is isometric to the product $(0,1] \times M$ in a collaring neighborhood of $M$; and

(b) a $(\mathbb{Z} / 2 \mathbb{Z}$-graded) Dirac bundle on $W$ that is isomorphic to the pullback of $S \oplus S$ over the collaring neighborhood, with action of TW on the collaring neighborhood given by

$$
c_{W}\left(\partial_{t}\right)=\left(\begin{array}{cc}
0 & -I \\
I & 0
\end{array}\right) \quad \text { and } \quad c_{W}(Y)=\left(\begin{array}{cc}
0 & c_{M}(Y) \\
c_{M}(Y) & 0
\end{array}\right)
$$

for all $Y \in T M$.

A Dirac bundle $S$ provides data sufficient to define an elliptic operator: there is a formally self-adjoint, elliptic first-order differential operator D acting on sections of $S$ such that for any smooth scalar function $\phi$,

$$
[\mathrm{D}, \phi]=\mathrm{c}(\operatorname{grad} \phi): \mathrm{S} \longrightarrow \mathrm{S} .
$$

3.5. Definition. We shall refer to any operator D, as above, as a Dirac operator for the geometric K-cycle $(M, S, f)$.

3.6. Remark. If the cycle $(M, S, f)$ is a boundary, then any Dirac operator for it is a boundary in the sense of Definition 2.1.

There is a natural operation of disjoint union on geometric K-cycles:

$$
\left(M^{\prime}, S^{\prime}, f^{\prime}\right) \sqcup\left(M^{\prime \prime}, S^{\prime \prime}, f^{\prime \prime}\right)=\left(M^{\prime} \sqcup M^{\prime \prime}, S^{\prime} \sqcup S^{\prime \prime}, f^{\prime} \sqcup f^{\prime \prime}\right) .
$$

We shall say that $\left(M^{\prime}, S^{\prime}, f^{\prime}\right)$ is a component of the disjoint union. We shall also define the negative of a geometric cycle $(M, S, f)$ to be the cycle

$$
-(M, S, f)=(M,-S, f),
$$

where $-\mathrm{S}$ denotes the bundle $\mathrm{S}$ with the Clifford action $-\mathrm{c}$.

3.7. Definition. Two geometric K-cycles are bordant if the disjoint union of one with the negative of the other is a boundary. 
Bordism is part of the equivalence relation among geometric K-cycles that defines K-homology. A second important part is an operation called bundle modification that relates manifolds of different dimensions. To describe it we need some preliminary definitions.

3.8. Definition. (Compare [10, Definition 3.10].) Denote by $\theta$ the self-adjoint involution of the Hermitian bundle Cliff( $\mathrm{N})$ of complex Clifford algebras associated to $\mathrm{TN}$ that is given by right multiplication by the element

$$
\theta=i^{k} e_{1} \cdots e_{2 k}
$$

of Cliff $(N)$. Here $e_{1}, \ldots, e_{2 k}$ is a local oriented orthonormal frame for $\mathbb{T N}$ ( $\theta$ does not depend on the choice of frame). Denote by

$$
\operatorname{Cliff}_{\theta}(\mathrm{N}) \subseteq \operatorname{Cliff}(\mathrm{N})
$$

the +1 eigenbundle for $\theta$, equipped with the Dirac bundle structure obtained from the natural $\mathbb{Z} / 2 \mathbb{Z}$-grading of $\operatorname{Cliff}(\mathrm{N})$ and the left multiplication action of $\mathrm{TN}$ on $\operatorname{Cliff}(\mathrm{N})$.

Now fix an orientation on the unit sphere $S^{2 k} \subseteq \mathbb{R}^{2 k+1}$ (it does not matter which one). The group $\mathrm{SO}(2 \mathrm{k})$ acts on $S^{2 \mathrm{k}}$ via the standard inclusion of $\mathrm{SO}(2 \mathrm{k})$ into $S O(2 k+1)$. In addition $S O(2 k)$ acts on $\operatorname{Cliff}_{\theta}\left(S^{2 k}\right)$ in such a way that the Clifford action

$$
c: \mathrm{TS}^{2 \mathrm{k}} \longrightarrow \operatorname{End}\left(\operatorname{Cliff}_{\theta}\left(\mathrm{S}^{2 \mathrm{k}}\right)\right)
$$

is $\mathrm{SO}(2 \mathrm{k})$-equivariant.

3.9. Definition. Let $(M, S, f)$ be a geometric K-cycle for $X$ and let $P$ be a smooth principal $\mathrm{SO}(2 \mathrm{k})$-bundle over $\mathrm{M}$. An elementary bundle modification of a cycle $(M, S, f)$ associated to the principal bundle $P$ is a geometric K-cycle $(\widehat{M}, \hat{S}, \hat{f})$, where:

(a) $\widehat{M}$ is the fibre bundle $\mathrm{P} \times{ }_{\mathrm{SO}(2 \mathrm{k})} \mathrm{S}^{2 \mathrm{k}}$, equipped with any Riemannian metric that agrees with the metric of $S^{2 k}$ on vertical tangent vectors and that agrees with the metric of $M$ on horizontal tangent vectors.

(b) $\hat{S}$ is the tensor product of the pullback to $\widehat{M}$ of $S$ with the bundle on $\widehat{M}$ induced from the $S O(2 k)$-equivariant bundle $\operatorname{Cliff}_{\theta}\left(S^{2 k}\right)$ on the sphere $S^{2 k}$. 
The action $\mathrm{c}: \mathrm{T} \hat{M} \rightarrow \operatorname{End}(\hat{\mathrm{S}})$ is given by the formula

$$
c(X)= \begin{cases}c(X) \otimes \varepsilon & \text { if } X \text { is horizontal } \\ I \otimes c(X) & \text { if } X \text { is vertical }\end{cases}
$$

where $\varepsilon$ is the grading operator on the bundle $\mathrm{P} \times{ }_{\mathrm{SO}(2 \mathrm{k})} \mathrm{Cliff}_{\theta}\left(\mathrm{S}^{2 \mathrm{k}}\right)$.

(c) $\hat{f}$ is the composition fo $\pi: \widehat{M} \rightarrow X$, where $\pi: \widehat{M} \rightarrow M$ is the natural projection.

3.10. Definition. Let $(M, S, f)$ be a geometric K-cycle for X. A bundle modification of $(M, S, f)$ is any cycle obtained by elementary bundle modification of a component of $(M, S, f)$.

3.11. Definition. Let $X$ be a space. We denote by $K_{1}^{\text {geom }}(X)$ the quotient of the space of geometric $\mathrm{K}$-cycles for $\mathrm{X}$ by the equivalence relation generated by the following three relations:

(a) Direct sum/disjoint union. The cycle $\left(M, S_{1} \oplus S_{2}, f\right)$ is equivalent to the disjoint union $\left(M, S_{1}, f\right) \sqcup\left(M, S_{2}, f\right)$.

(b) Bordism. Bordant cycles are equivalent.

(c) Bundle Modification. A cycle is equivalent to any cycle that is obtained from it by bundle modification.

We refer to [8], [10] or [26] for further details. The straightforward isomorphism between Baum's K-homology groups and those defined here is described in [26].

\section{Analytic K-Homology}

Although we shall be mostly concerned with geometric K-homology in this paper, it will be convenient to work with the analytic definition of K-homology as well. We shall review the definition in this section.

In an important paper [1], Atiyah proposed a functional-analytic definition of K-homology. This was based on what we shall call an (odd) analytic K-cycle for a compact metrizable space $\mathrm{X}$, which consists of:

(a) A Hilbert space $\mathrm{H}$ equipped with a representation* of the $\mathrm{C}^{*}$-algebra $\mathrm{C}(\mathrm{X})$ of continuous functions on $\mathrm{X}$ as bounded operators on $\mathrm{H}$.

${ }^{*}$ Throughout, we shall assume that our representations are nondegenerate, which means that the constant function 1 on $\mathrm{X}$ acts as the identity operator on $\mathrm{H}$. 
(b) A self-adjoint Fredholm operator $\mathrm{F}$ on $\mathrm{H}$ such that $\mathrm{F}^{2}=\mathrm{I}$, modulo compact operators, and such that $[F, f]=0$, modulo compact operators, for every $f \in C(X)$.

(For the even K-homology group there are additional conditions involving $\mathbb{Z} / 2 \mathbb{Z}$ gradings.) The equivalence relation between analytic K-cycles is a suitable notion of homotopy, and was worked out in detail by Kasparov [25] and by Brown, Douglas and Fillmore [12].

4.1. Example. If $D$ is a Dirac operator for a geometric K-cycle $(M, S, f)$ for $X$, then the operator $F=\operatorname{sign}(D)$ on $L^{2}(M, S)$ is an analytic K-cycle for $X$ (the map $f$ determines a representation of $C(X)$ on $L^{2}(M, S)$ ).

It will be convenient for us to view the K-homology groups of $\mathrm{X}$ as the $\mathrm{K}$ theory groups of a certain $C^{*}$-algebra associated to $X$. This is an approach that was originally suggested by Paschke [31], and then developed by the authors in [19] and [21]. To this end, let us consider a Hilbert space $H$ that is equipped with a representation of the $\mathrm{C}^{*}$-algebra $\mathrm{C}(\mathrm{X})$ as bounded operators. We define

$$
\mathrm{D}_{\mathrm{H}}^{*}(\mathrm{X})=\{\mathrm{T} \in \mathrm{B}(\mathrm{H}):[\mathrm{T}, \mathrm{f}]=0 \text { modulo compact operators, } \forall \mathrm{f} \in \mathrm{C}(\mathrm{X})\}
$$

and

$$
\mathrm{Q}_{\mathrm{H}}^{*}(\mathrm{X})=\text { Quotient of } \mathrm{D}_{\mathrm{H}}^{*}(\mathrm{X}) \text { by the ideal of compact operators on } \mathrm{H} \text {. }
$$

An analytic K-cycle determines a projection in the quotient $C^{*}$-algebra $Q_{H}^{*}(X)$ via the formula $P=\frac{1}{2}(F+1)$, and conversely, any projection in $Q_{H}^{*}(X)$ determines an analytic K-cycle by a reversal of this process.

If $\mathrm{H}_{1} \subseteq \mathrm{H}$ is a subspace and subrepresentation of $\mathrm{C}(\mathrm{X})$, then there are inclusions

$$
\mathrm{D}_{\mathrm{H}_{1}}^{*}(\mathrm{X}) \longrightarrow \mathrm{D}_{\mathrm{H}}^{*}(\mathrm{X}) \text { and } \mathrm{Q}_{\mathrm{H}_{1}}^{*}(\mathrm{X}) \longrightarrow \mathrm{Q}_{\mathrm{H}}^{*}(\mathrm{X})
$$

given by extending any operator on $\mathrm{H}_{1}$ to an operator on $\mathrm{H}$ that is zero on the orthogonal complement of $\mathrm{H}_{1}$. If $\mathrm{H}_{1}$ is ample, in the sense that no nonzero function in $\mathrm{C}(\mathrm{X})$ acts as a compact operator on $\mathrm{H}_{1}$, then these inclusions induce isomorphisms at the level of K-theory. In particular, the $\mathrm{C}^{*}$-algebras associated to two ample Hilbert spaces have canonically isomorphic K-theory groups.

This prompts us to define $K_{*}\left(D^{*}(X)\right)$ (the subscript $H$ has been dropped) to be the K-theory of $\mathrm{D}_{\mathrm{H}}^{*}(\mathrm{X})$ for any ample $\mathrm{H}$. We define $\mathrm{K}_{*}\left(\mathrm{Q}^{*}(\mathrm{X})\right)$ in the same way. If 
$\mathrm{f}: \mathrm{X} \rightarrow \mathrm{Y}$ is a continuous map, and if $\mathrm{H}$ is equipped with a representation of $\mathrm{C}(\mathrm{X})$, then denote by $f_{*} \mathrm{H}$ the same Hilbert space equipped with the representation of $\mathrm{C}(\mathrm{Y})$ associated to the homomorphism $\mathrm{C}(\mathrm{Y}) \rightarrow \mathrm{C}(\mathrm{X})$ induced from $\mathrm{f}$. There are inclusions

$$
\mathrm{D}_{\mathrm{H}}^{*}(\mathrm{X}) \longrightarrow \mathrm{D}_{\mathrm{f}_{*} \mathrm{H}}^{*}(\mathrm{Y}) \text { and } \mathrm{Q}_{\mathrm{H}}^{*}(\mathrm{X}) \longrightarrow \mathrm{Q}_{\mathrm{f}_{*} \mathrm{H}}^{*}(\mathrm{Y})
$$

By including $f_{*} \mathrm{H}$ into an ample Hilbert space for $\mathrm{Y}$, we functorially associate to the map $f: X \rightarrow Y$ homomorphisms

$$
f_{*}: K_{*}\left(D^{*}(X)\right) \rightarrow K_{*}\left(D^{*}(Y)\right) \text { and } f_{*}: K_{*}\left(Q^{*}(X)\right) \rightarrow K_{*}\left(Q^{*}(Y)\right) .
$$

With these observations in mind we now define

$$
\mathrm{K}_{1}(\mathrm{X}) \cong \mathrm{K}_{0}\left(\mathrm{Q}^{*}(\mathrm{X})\right) \text { and } \mathrm{K}_{0}(\mathrm{X}) \cong \mathrm{K}_{1}\left(\mathrm{Q}^{*}(\mathrm{X})\right) .
$$

These are the analytic K-homology groups of Atiyah, Kasparov, and BrownDouglas-Fillmore. See [21] for a detailed treatment.

4.2. Example. The arguments in [10] show that the analytic K-homology class that we associated to a geometric K-cycle $(M, S, f)$ in Example 4.1 depends only on the class of $(M, S, f)$ in geometric K-homology. It is also shown in [10] that the map

$$
\mathrm{K}_{1}^{\text {geom }}(\mathrm{X}) \longrightarrow \mathrm{K}_{1}(\mathrm{X})
$$

thereby defined is an isomorphism, as long as $\mathrm{X}$ is a finite $\mathrm{CW}$-complex.

4.3. Example. An analytic cycle is degenerate if the operator F commutes with the action of $\mathrm{C}(\mathrm{X})$. A simple argument shows that any degenerate cycle defines the zero element in K-homology (compare [21, Proposition 8.2.8]).

We shall need to deal with analytic K-homology for certain more general spaces, specifically for $\mathrm{CW}$-complexes that are equipped with the $\mathrm{CW}$-topology, in which a subset is closed if and only if its intersection with each finite subcomplex is a closed subset of that subcomplex. We shall do so as follows:

4.4. Definition. Let $W$ be a $C W$ complex with the $C W$-topology. We define

$$
K_{*}(W)=\underset{X \subseteq W}{\lim _{X \subseteq W}} K_{*}(X)
$$

where the limit is over the finite subcomplexes of $W$. 
4.5. Example. If $f: M \rightarrow W$ is any map from a compact space into a $C W$ complex, then the image of $f$ is contained within a finite subcomplex of $W$, and it therefore follows that

$$
K_{1}^{\text {geom }}(W) \cong \underset{X \subseteq W}{\lim _{X}} K_{1}^{\text {geom }}(X)
$$

with the direct limit being again over finite subcomplexes. As a result, the isomorphism from geometric to analytic K-homology for finite CW-complexes extends to an isomorphism for arbitrary CW-complexes.

\section{The Assembly MaP}

In this section we shall review the definitions of the assembly map and the analytic structure groups $\mathcal{S}_{*}(\pi)$ that measure how far the assembly map is from an isomorphism.

We shall stray from current custom in $\mathrm{C}^{*}$-algebra $\mathrm{K}$-theory by defining the assembly map to be a homomorphism into the K-theory of the full group $\mathrm{C}^{*}$-algebra, as opposed to the reduced group $\mathrm{C}^{*}$-algebra. This was the original approach of Kasparov, but it is not the approach followed by Baum and Connes. Our definition of the structure groups $\mathcal{S}_{*}(\pi)$ will be similarly adapted to the full group $\mathrm{C}^{*}$-algebra.

We shall need to begin with some background material on Hilbert modules. For detailed information on this topic the reader is referred to the monograph [28].

A Hilbert module over a $C^{*}$-algebra $A$ is a Banach space $H$ equipped with a right $A$-action by bounded operators as well as an $A$-valued inner product that is related to the Banach space norm by the formula

$$
\|s\|_{\mathrm{H}}^{2}=\|\langle s, s\rangle\|_{\mathrm{A}} \quad \forall s \in \mathrm{H} .
$$

A Hilbert module over $A=\mathbb{C}$ is just a Hilbert space.

5.1. Example. The space $\Gamma(V)$ of continuous sections of a Hermitian vector bundle over a compact space $\mathrm{X}$ is a Hilbert module over $\mathrm{C}(\mathrm{X})$ (the inner product is given by pointwise inner product of sections). 
If $\mathrm{E}$ is a Hilbert module over a $\mathrm{C}^{*}$-algebra $\mathrm{B}$, and if $\mathrm{H}$ is a Hilbert module over A that is also equipped with a left action of B by bounded, adjointable operators, ${ }^{\dagger}$ then we may form the Hilbert module interior tensor product $\mathrm{E} \otimes_{\mathrm{B}} \mathrm{H}$, which is a Hilbert A-module completion of the algebraic tensor product. Its inner product is given by the formula

$$
\left\langle e_{1} \otimes v_{1}, e_{2} \otimes v_{2}\right\rangle=\left\langle v_{1},\left\langle e_{1}, e_{2}\right\rangle v_{2}\right\rangle .
$$

See see [28, page 41].

5.2. Example. If $A=\mathbb{C}$ and $B=C(M)$, if $V$ is a Hermitian vector bundle over $X$, and if $\mathrm{H}$ is the Hilbert space of $\mathrm{L}^{2}$-sections of some other Hermitian bundle $\mathrm{S}$ over $X$, then $\Gamma(V) \otimes_{B} H$ is the Hilbert space of $L^{2}$-sections of $V \otimes S$.

Suppose that $\mathrm{T}$ is a bounded operator on $\mathrm{H}$. In situations like the one in Example 5.2 we should like to form the tensor product operator $I \otimes T$ on $E \otimes_{B} H$. For example we shall need to lift operators acting on sections of some bundle $S$ to operators acting on sections of a tensor product bundle $E \otimes S$. But unless $T$ commutes with the action of $B$, the operator $I \otimes T$ on $E \otimes_{B} H$ will not be well-defined.

The problem can be addressed as follows. First, a bounded operator between Hilbert modules over $A$ is said to be compact if it is a norm-limit of finite linear combinations of elementary operators of the form $v \mapsto v_{1}\left\langle v_{2}, v\right\rangle$. If $A=\mathbb{C}$, then this is the standard notion of compact operator on Hilbert space.

Next, for each element $e \in \mathrm{E}$, define an operator

$$
\mathrm{L}_{e}: \mathrm{H} \rightarrow \mathrm{E} \otimes_{\mathrm{B}} \mathrm{H}
$$

by the formula $L_{e}(v)=e \otimes v$.

5.3. Definition. Let $E$ be a Hilbert module over a $C^{*}$-algebra $B$ and let $\mathrm{H}$ be a Hilbert module over $A$ that is also equipped with a left action of $B$ by bounded operators. Let $\mathrm{T}$ be a bounded operator on $\mathrm{H}$ that commutes with the given action of $B$, modulo compact Hilbert module operators. An operator $T_{E}: E \otimes_{B} H \rightarrow$

\footnotetext{
${ }^{\dagger}$ Unlike the case of Hilbert spaces, operators on Hilbert modules, even bounded A-linear ones, need not have adjoints in general. Those that do are called adjointable, and we shall work exclusively with them.
} 
$\mathrm{E} \otimes_{\mathrm{B}} \mathrm{H}$ is a lift of $\mathrm{T}$ if for every $e \in \mathrm{E}$ the diagrams

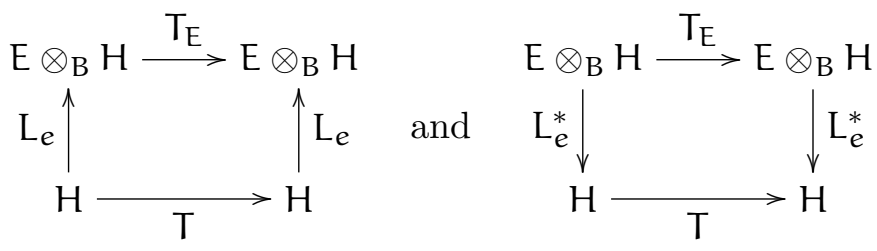

commute modulo compact Hilbert module operators.

This concept of a lift of an operator is borrowed from $[14,35]$, where the term connection is used.

5.4. Definition. We shall say that a Hilbert module over a unital $C^{*}$-algebra is finitely generated or projective if the underlying algebraic module has the corresponding property. ${ }^{\ddagger}$

The following lemma is easily proved by embedding $\mathrm{E}$ into a free module (compare [14, Appendix A]).

5.5. Lemma. If $\mathrm{E}$ is a finitely generated and projective Hilbert module over a unital $\mathrm{C}^{*}$-algebra, then every operator $\mathrm{T}$ as in Definition 5.3 has a lift, and any two lifts differ by a compact operator. Furthermore $\mathrm{T}$ lifts to a compact operator if and only if it is itself compact.

5.6. Example. Let D be a first order self-adjoint elliptic partial differential operator acting on sections of a smooth Hermitian bundle $S$ over a closed manifold $M$, and let $\mathrm{V}$ be a second Hermitian bundle on $\mathrm{M}$. If we fix a Hermitian connection on $\mathrm{V}$, then, as is well known, we may use the connection to cause $\mathrm{D}$ to act as an elliptic operator $D_{V}$ on the smooth sections of $V \otimes S$. If we set $F=\operatorname{sign}(D)$ and $F_{V}=\operatorname{sign}\left(D_{V}\right)$, then $F_{V}$ is a lift of $F$ in the sense of Definition 5.3.

Now, fix a countable group $\pi$. Recall that $C^{*}(\pi)$ is a $C^{*}$-algebra completion of the complex group algebra. It has the universal property that for any embedding of $\pi$ into the unitary group of a $C^{*}$-algebra $A$ there is a unique $*$-homomorphism

\footnotetext{
${ }^{\ddagger}$ It happens that finite generation actually implies projectivity for Hilbert modules, but we shall not use this fact.
} 
from $C^{*}(\pi)$ to $A$ such that the diagram

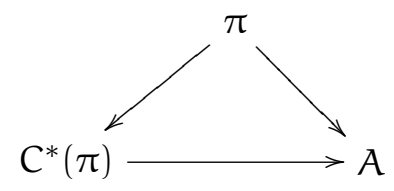

commutes.

Fix a classifying space $B \pi$ for the group $\pi$. We can and shall choose $B \pi$ to be a $C W$-complex with the $C W$-topology. In addition, fix a map $f: X \rightarrow B \pi$ and so a principal $\pi$-space $\widetilde{X}$ over $X$.

5.7. Definition. The Mishchenko line bundle over X is the quotient space

$$
\mathrm{L}=\tilde{\mathrm{X}} \times_{\pi} \mathrm{C}^{*}(\pi)
$$

by the diagonal left action of $\pi$.

The Mishchenko line bundle is an example of a vector $C^{*}(\pi)$-bundle, in the sense of the following definition.

5.8. Definition. Let $A$ be a unital $C^{*}$-algebra and let $X$ be a topological space. A vector $A$-bundle is a locally trivial bundle over $\mathrm{X}$ whose fibers are finitely generated and projective Hilbert modules over $A$, and whose structural group is the group of unitary Hilbert module operators, equipped with the norm topology.

5.9. Remark. In fact the Mishchenko line bundle has the additional structure of a flat vector $C^{*}(\pi)$-bundle. Its space of sections is

$$
\Gamma(\mathrm{L}) \cong\left\{\mathrm{s}: \tilde{\mathrm{X}} \stackrel{\text { continuous }}{\longrightarrow} \mathrm{C}^{*}(\pi): \mathrm{s}(\mathrm{g} \tilde{\mathrm{x}})=\mathrm{gs}(\tilde{\mathrm{x}}) \quad \forall \mathrm{g} \in \pi \quad \forall \tilde{\mathrm{x}} \in \tilde{\mathrm{X}}\right\}
$$

and in this description a locally defined section is parallel if it is locally constant.

If $X$ is compact, then the space of continuous sections of any vector $A$-bundle is a finitely generated and projective module over the $C^{*}$-algebra $C(X, A)$ of continuous, $A$-valued functions on $X$. Now the $C^{*}$-algebra $C(X, A)$ is isomorphic to the tensor product $C(X) \otimes A$, and switching to the special case $A=C^{*}(\pi)$ this allows us to adjust the concept of lift introduced in Definition 5.3, as follows.

Let $\mathrm{H}$ be a Hilbert space that is equipped with a representation of $\mathrm{C}(\mathrm{X})$. Form the Hilbert module exterior tensor product $\mathrm{H} \otimes \mathrm{C}^{*}(\pi)$. This is a Hilbert module 
completion of the algebraic tensor product over $\mathbb{C}$. $\operatorname{Its} C^{*}(\pi)$-valued inner product is given by the formula

$$
\left\langle v_{1} \otimes f_{1}, v_{2} \otimes f_{2}\right\rangle=\left\langle v_{1}, v_{2}\right\rangle f_{1}^{*} f_{2} .
$$

See $\left[28\right.$, page 35]. The exterior tensor product has a left action of $C(X) \otimes C^{*}(\pi)$ by bounded operators, and so we can form the tensor product

$$
\mathrm{H}_{\pi}=\Gamma(\mathrm{L}) \otimes_{\mathrm{C}(\mathrm{X}) \otimes \mathrm{C}^{*}(\pi)}\left(\mathrm{H} \otimes \mathrm{C}^{*}(\pi)\right) .
$$

We shall say that an operator $\mathrm{T}_{\pi}$ on $\mathrm{H}_{\pi}$ is a lift of an operator $\mathrm{T} \in \mathrm{D}_{\mathrm{H}}^{*}(\mathrm{X})$ if $\mathrm{T}_{\pi}$ is a lift of the operator $\mathrm{T} \otimes \mathrm{I}$ on $\mathrm{H} \otimes \mathrm{C}^{*}(\pi)$ in the sense of Definition 5.3.

5.10. Example. If $\mathrm{H}$ is the space of $\mathrm{L}^{2}$-sections of a vector bundle $\mathrm{S}$ over $\mathrm{X}$ (with respect to some measure $\mu$ on $X$ ), then $H_{\pi}$ is the space of $L^{2}$-sections of the $C^{*}(\pi)$-vector bundle formed from the tensor product of $S$ with the Mischenko line bundle (that is, $\mathrm{H}_{\pi}$ is the completion of the space of continuous sections of this tensor product bundle in the natural Hilbert module norm associated to $\mu$ ). We shall discuss explicit examples of liftings in this case in Section 7.

We can now give the definitions of the assembly map and of the analytic structure groups.

5.11. Definition. Denote by $\mathrm{D}_{\mathrm{H}_{\pi}}^{*}(\mathrm{X})$ the algebra of operators on $\mathrm{H}_{\pi}$ that are lifts of operators in $\mathrm{D}_{\mathrm{H}}^{*}(\mathrm{X})$, and denote by $\mathrm{Q}_{\mathrm{H}_{\pi}}^{*}(\mathrm{X})$ the quotient of $\mathrm{D}_{\mathrm{H}_{\pi}}^{*}(\mathrm{X})$ by the ideal $\mathcal{K}\left(\mathrm{H}_{\pi}\right)$ of compact Hilbert module operators on $\mathrm{H}_{\pi}$.

Since, as noted in Lemma 5.5, every T has a lifting, and since any two liftings are equal, modulo compact operators, and since a lifting of $\mathrm{T}$ is compact Hilbert module operator if and only if $\mathrm{T}$ is a compact Hilbert space operator, it follows that $\mathrm{Q}_{\mathrm{H}_{\pi}}^{*}(\mathrm{X})$ is isomorphic to $\mathrm{Q}_{\mathrm{H}}^{*}(\mathrm{X})$, and that there is a short exact sequence

$$
0 \longrightarrow \mathcal{K}\left(\mathrm{H}_{\pi}\right) \longrightarrow \mathrm{D}_{\mathrm{H}_{\pi}}^{*}(\mathrm{X}) \longrightarrow \mathrm{Q}_{\mathrm{H}}^{*}(\mathrm{X}) \longrightarrow 0
$$

The $\mathrm{C}^{*}$-algebra of compact operators on $\mathrm{H}_{\pi}$ is canonically Morita equivalent to $C^{*}(\pi)$, at least as long as $H \neq 0$. This is a general fact about Hilbert $A$-modules with the property that the space of inner products $\left\langle v_{1}, v_{2}\right\rangle$ generates $A$. There is therefore a canonical isomorphism

$$
\mathrm{K}_{*}\left(\mathcal{K}\left(\mathrm{H}_{\pi}\right)\right) \cong \mathrm{K}_{*}\left(\mathrm{C}^{*}(\pi)\right) .
$$


The K-theory groups $\mathrm{K}_{*}\left(\mathrm{D}_{\mathrm{H}_{\pi}}^{*}(\mathrm{X})\right)$ are independent of the choice of Hilbert space $\mathrm{H}$, as long as it is ample. They are covariantly functorial on the category of compact metrizable spaces that are equipped with maps to $\mathrm{B} \pi$ (compare the discussion in Section 4 about the K-theory groups $\mathrm{K}_{*}\left(\mathrm{D}_{\mathrm{H}}^{*}(\mathrm{X})\right)$ ). From the abovedisplayed short exact sequence of $\mathrm{C}^{*}$-algebras we obtain a long exact sequence of the form

$$
\begin{aligned}
\cdots \longrightarrow \mathrm{K}_{0}(X) \stackrel{\mu}{\longrightarrow} \mathrm{K}_{0}\left(\mathrm{C}^{*}(\pi)\right) \longrightarrow \mathrm{K}_{0}\left(\mathrm{D}_{\pi}^{*}(\mathrm{X})\right) & \\
& \longrightarrow \mathrm{K}_{1}(\mathrm{X}) \stackrel{\mu}{\longrightarrow} \mathrm{K}_{1}\left(\mathrm{C}^{*}(\pi)\right) \longrightarrow \cdots
\end{aligned}
$$

As in our earlier definition of K-homology, we have dropped mention of the Hilbert space $\mathrm{H}$.

The maps labelled $\mu$ are the connecting maps in the K-theory exact sequence. These are the assembly maps (for even and odd K-homology) associated to the map $f: X \rightarrow B \pi$, as introduced by Kasparov in connection with the Novikov conjecture [25].

5.12. Example. Let $(M, S, f)$ be a geometric K-cycle for $B \pi$ and let $D$ be a Dirac operator. Let us calculate the image of its K-homology class under the assembly map.

As is well known, the connecting homomorphism maps the class of a projection $p$ in the $K_{0}$-group of a quotient $C^{*}$-algebra $A / J$ to the class of the unitary operator $\exp (2 \pi i \tilde{p})$ in $K_{1}(J)$, where $\tilde{p}$ is any self-adjoint lift of $p$. We find that

$$
\mu([M, S, f])=\left[\exp \left(2 \pi i \cdot \frac{1}{2}\left(F_{\pi}+I\right)\right)\right]
$$

where $F_{\pi}$ is any lift of the operator $F=\operatorname{sign}(D)$ on $H=L^{2}(M, S)$.

As we noted in Example 5.10, the Hilbert module $\mathrm{H}_{\pi}$ is the space of $\mathrm{L}^{2}$ sections of the vector $C^{*}(\pi)$-bundle $L \otimes S$, where $L$ is the Mishchenko line bundle. Moreover we noted in Remark 5.9, the Mishchenko line bundle carries a natural flat connection, and we may use it to cause $D$ to act as an operator $D_{\pi}$ on $L \otimes S .{ }^{\S}$ We would like to follow Example 5.6 to define an explicit lift of $F$. There is however a crucial detail that must be attended to: the spectrum of $D_{\pi}$ is not discrete, and it is therefore not generally possible to define $F_{\pi}=\operatorname{sign}\left(D_{\pi}\right)$. Indeed, if we could, then the above-displayed exponential would be the identity operator. However

\footnotetext{
${ }^{\S}$ Using the identification of $\Gamma(\mathrm{L})$ given in Remark 5.9 , we get $\mathrm{D}_{\pi}=\mathrm{I} \otimes \mathrm{D}$.
} 
it is possible to apply to $\mathrm{D}_{\pi}$ any bounded continuous function on the spectrum (see for example [34]), and so for example form the operator

$$
\mathrm{F}_{\pi}=\mathrm{D}_{\pi}\left(\mathrm{I}+\mathrm{D}_{\pi}^{2}\right)^{-\frac{1}{2}} \quad \text { or } \quad \mathrm{F}_{\pi}=\frac{2}{\pi} \arctan \left(\mathrm{D}_{\pi}\right)
$$

Both of the displayed functions agree with $\operatorname{sign}(x)$ modulo a function that vanishes at infinity. As a result the corresponding operators $F_{\pi}$ differ by a compact operator and they both lift $F$. Using the second definition of $F_{\pi}$ we find that

$$
\exp \left(2 \pi i \cdot \frac{1}{2}\left(F_{\pi}+I\right)\right)=\left(D_{\pi}-i I\right)\left(D_{\pi}+i I\right)^{-1}
$$

because $\exp (2 i \arctan (x))=-(x-i)(x+i)^{-1}$. We conclude that

$$
\mu([M, S, f])=\left[\left(D_{\pi}-i I\right)\left(D_{\pi}+i I\right)^{-1}\right] .
$$

Thus image of a geometric cycle under the assembly map is the K-theory class determined by the Cayley transform of the Dirac operator $\mathrm{D}_{\pi}$.

We conclude by defining the analytic structure groups.

5.13. Definition. Denote by $\mathcal{S}_{1}(\pi)$ the direct limit

$$
\mathcal{S}_{1}(\pi)=\underset{X \subseteq B \pi}{\lim } \mathrm{K}_{0}\left(D_{\pi}^{*}(X)\right),
$$

over finite subcomplexes of the classifying space $B \pi$.

We can define $\mathcal{S}_{0}(\pi)$ similarly, using the other K-theory group, but we shall not need to use $\mathcal{S}_{0}(\pi)$ in this paper. The long exact sequence given above now translates into the following analytic surgery exact sequence for the group $\pi$ :

$$
\begin{aligned}
\cdots \longrightarrow \mathrm{K}_{0}(\mathrm{~B} \pi) \stackrel{\mu}{\longrightarrow} \mathrm{K}_{0}\left(\mathrm{C}^{*}(\pi)\right) & \longrightarrow \mathcal{S}_{1}(\pi) \\
& \longrightarrow \mathrm{K}_{1}(\mathrm{~B} \pi) \stackrel{\mu}{\longrightarrow} \mathrm{K}_{1}\left(\mathrm{C}^{*}(\pi)\right) \longrightarrow \cdots .
\end{aligned}
$$

5.14. Remark. There is clearly a more general surgery exact sequence for any map $f: X \rightarrow B \pi$. It takes the form

$$
\begin{aligned}
\cdots \longrightarrow \mathrm{K}_{0}(\mathrm{X}) \stackrel{\mu}{\longrightarrow} \mathrm{K}_{0}\left(\mathrm{C}^{*}(\pi)\right) \longrightarrow \mathcal{S}_{1}(\mathrm{f}: \mathrm{X} \rightarrow \mathrm{B} \pi) \\
\longrightarrow \mathrm{K}_{1}(\mathrm{X}) \stackrel{\mu}{\longrightarrow} \mathrm{K}_{1}\left(\mathrm{C}^{*}(\pi)\right) \longrightarrow \cdots,
\end{aligned}
$$

where the structure groups are the K-theory groups of $D_{\pi}^{*}(X)$. This is the counterpart for $\mathrm{C}^{*}(\pi)$ of the general sequence considered in $[22,23,24]$. We shall not need the more general sequence in this paper. 


\section{The $\mathbb{R} / \mathbb{Z}$-IndeX}

Fix a group $\pi$ and a classifying space $B \pi$ as in the previous section. In addition, fix two group homomorphisms $\sigma_{1}, \sigma_{2}: \pi \rightarrow U(N)$. Our main purpose in this section is to return to the definition of geometric K-homology and prove the following compatibility with relative eta invariants:

6.1. Theorem. Let $(\mathrm{M}, \mathrm{S}, \mathrm{f})$ be a geometric $\mathrm{K}$-cycle for $\mathrm{B} \pi$ and let $\mathrm{D}$ be a Dirac operator for $(\mathrm{M}, \mathrm{S}, \mathrm{f})$. The quantity

$$
\rho\left(D, f, \sigma_{1}, \sigma_{2}\right) \quad \bmod \mathbb{Z}
$$

depends only on the equivalence class of $(M, S, f)$ in $\mathrm{K}_{1}(\mathrm{~B} \pi)$.

We need to analyze the three relations that generate the equivalence relation on cycles in geometric K-homology.

6.2. Lemma. If the geometric $\mathrm{K}$-cycle $(\mathrm{M}, \mathrm{S}, \mathrm{f})$ for $\mathrm{B} \pi$ is a boundary, and if $\mathrm{D}$ is any Dirac operator for $(M, S, f)$, then the relative eta invariant $\rho\left(D, f, \sigma_{1}, \sigma_{2}\right)$ is an integer.

Proof. This follows immediately from Lemma 2.4, since D is a boundary in the sense of Definition 2.1, as noted in Remark 3.6.

6.3. Proposition. If $\left(\mathrm{M}^{\prime}, \mathrm{S}^{\prime}, \mathrm{f}^{\prime}\right)$ and $\left(\mathrm{M}^{\prime \prime}, \mathrm{S}^{\prime \prime}, \mathrm{f}^{\prime \prime}\right)$ are geometric $\mathrm{K}$-cycles for $\mathrm{B} \pi$ that are bordant, and if $\mathrm{D}^{\prime}$ and $\mathrm{D}^{\prime \prime}$ are Dirac operators for these cycles, then the relative eta invariants $\rho\left(D^{\prime}, f^{\prime}, \sigma_{1}, \sigma_{2}\right)$ and $\rho\left(D^{\prime \prime}, f^{\prime \prime}, \sigma_{1}, \sigma_{2}\right)$ are equal, modulo integers.

Proof. If D is any Dirac operator, then it is clear that the relative eta invariant for $-\mathrm{D}$ is minus the relative eta invariant for $\mathrm{D}$. Moreover the relative eta invariant for a Dirac operator on a disjoint union of manifolds is the sum of the relative eta invariants of the operators on the components making up the disjoint union. The proposition therefore follows by applying the previous lemma to the disjoint union cycle

$$
-\left(M^{\prime}, S^{\prime}, f^{\prime}\right) \sqcup\left(M^{\prime \prime}, S^{\prime \prime}, f^{\prime \prime}\right)
$$

which is a boundary.

6.4. Corollary. The relative eta invariant $\rho\left(D, f, \sigma_{1}, \sigma_{2}\right)$, modulo $\mathbb{Z}$, is independent of the choice of Dirac operator D associated to a cycle (M, S, f). 
6.5. Lemma. Two cycles for $\mathrm{B} \pi$ that are equivalent by the direct sum/disjoint union relation have the same relative eta invariants, modulo $\mathbb{Z}$.

Proof. This is clear.

It remains to consider bundle modification. We shall show that two cycles that are equivalent via an elementary bundle modification have the same relative eta invariant. This will (more than) suffice. If D is a Dirac operator, then define the multiplicity function of $\mathrm{D}$ to be

$$
\operatorname{mult}_{D}(\lambda)=\text { Multiplicity of } \lambda \text { as an eigenvalue of } D \text {. }
$$

The eta invariant of $\mathrm{D}$ depends only on its multiplicity function. Moreover if $\mathrm{D}^{\prime}$ and $D^{\prime \prime}$ are two Dirac operators, and if their multiplicity functions are equivalent in the sense that the difference mult $\mathrm{D}^{\prime}-$ mult $_{\mathrm{D}^{\prime \prime}}$ is an even function that vanishes at $\lambda=0$, then the eta invariants of $\mathrm{D}^{\prime}$ and $\mathrm{D}^{\prime \prime}$ are equal to one another. We shall prove the following result:

6.6. Proposition. Let $(\mathrm{M}, \mathrm{S}, \mathrm{f})$ be an geometric $\mathrm{K}$-cycle for $\mathrm{B} \pi$. If the cycle $(\widehat{M}, \hat{S}, \hat{f})$ is obtained from $(\mathrm{M}, \mathrm{S}, \mathrm{f})$ by an elementary bundle modification, then there are Dirac operators $\mathrm{D}$ and $\widehat{\mathrm{D}}$ for $(\mathrm{M}, \mathrm{S}, \mathrm{f})$ and $(\widehat{\mathrm{M}}, \hat{\mathrm{S}}, \hat{\mathrm{f}})$ such that for every homomorphism $\sigma: \mathrm{G} \rightarrow \mathrm{U}(\mathrm{N})$ the multiplicity functions of $\mathrm{D}_{\sigma}$ and $\widehat{\mathrm{D}}_{\sigma}$ are equivalent.

Before beginning the proof we need to record a key fact about the Dirac bundle $\operatorname{Cliff}_{\theta}\left(S^{2 k}\right)$ that was introduced in Section 3.

6.7. Lemma. ([10, Proposition 3.11].) There is an $\mathrm{SO}(2 \mathrm{k})$-equivariant Dirac operator for $\mathrm{Cliff}_{\theta}\left(\mathrm{S}^{2 \mathrm{k}}\right)$ whose kernel is a copy of the one-dimensional trivial representation of $\mathrm{SO}(2 \mathrm{k})$, concentrated in grading degree zero.

Proof of Proposition 6.6. The argument that follows is almost exactly the same as the one used in [10, Proposition 3.6] to prove that geometric cycles that are equivalent under bundle modification give rise to the same elements of analytic K-homology.

Let us first consider the case where principal bundle $\mathrm{P}$ from which the bundle modification is built is the trivial bundle $M \times S O(2 k)$. In this case $\widehat{M}$ is the direct 
product $M \times S^{2 k}$. The bundle $\hat{S}$ is the tensor product of the bundle $S$ over $M$ with the bundle $\operatorname{Cliff}_{\theta}\left(S^{2 k}\right)$ over the sphere, and the formula

$$
\hat{\mathrm{D}}=\mathrm{D} \otimes \varepsilon+\mathrm{I} \otimes \mathrm{D}_{\theta},
$$

in which $D$ is any Dirac operator for $(M, S, f)$ and $D_{\theta}$ is the Dirac operator of Lemma 6.7 , defines a Dirac operator for $(\widehat{M}, \widehat{S}, \hat{f})$. Now let

$$
J=I \otimes i \varepsilon \operatorname{sign}\left(D_{\theta}\right) .
$$

This is a self-adjoint operator that anticommutes with $\hat{D}$. Its square is the orthogonal projection $\mathrm{P}$ onto the orthogonal complement of the subspace

$$
\mathrm{L}^{2}(\mathrm{M}, \mathrm{S}) \otimes \operatorname{ker}\left(\mathrm{D}_{\theta}\right) \subseteq \mathrm{L}^{2}(\mathrm{M}, \mathrm{S}) \otimes \mathrm{L}^{2}\left(\mathrm{~S}^{2 \mathrm{k}}, \operatorname{Cliff}_{\theta}\left(\mathrm{S}^{2 \mathrm{k}}\right)\right) .
$$

The projection $\mathrm{P}$ commutes with $\widehat{\mathrm{D}}$, and so we can write

$$
\widehat{\mathrm{D}}=\mathrm{P}^{\perp} \hat{\mathrm{DP}} \mathrm{P}^{\perp}+\mathrm{P} \hat{\mathrm{DP}} \text {. }
$$

Since $\operatorname{ker}\left(\mathrm{D}_{\theta}\right)$ is one-dimensional and concentrated in degree zero, the first operator, on $L^{2}(M, S) \otimes \operatorname{ker}\left(D_{\theta}\right)$, is a copy of $D$. As for the second operator, if $v$ is an eigenvector for $\widehat{D}$ with eigenvalue $\lambda$ and $\mathrm{P} v=v$, then

$$
\widehat{\mathrm{D} J} v=-\mathrm{J} \hat{\mathrm{v}} v=-\lambda \mathrm{J} v
$$

and of course $\mathrm{PJ} v=\mathrm{J} v$. Thus the multiplicity function for $\widehat{\mathrm{D}}$ is the sum of the multiplicity function for $\mathrm{D}$ (coming from $\mathrm{P}^{\perp} \hat{\mathrm{D}} \mathrm{P}^{\perp}$ ) and an even function (the multiplicity function of P(̂P) that vanishes at zero.

The same argument can now be repeated for any twisting of $\mathrm{D}$ and $\widehat{\mathrm{D}}$, since

$$
\hat{\mathrm{D}}_{\sigma}=\mathrm{D}_{\sigma} \otimes \varepsilon+\mathrm{I} \otimes \mathrm{D}_{\theta}
$$

and this completes the proof of the proposition in the case where $\mathrm{P}$ is the trivial principal bundle.

In the general case, where the principal bundle $\mathrm{P}$ is nontrivial, we begin by noting that

$$
\mathrm{L}^{2}(\widehat{M}, \widehat{S}) \cong\left[\mathrm{L}^{2}\left(\mathrm{P}, \pi^{*} \mathrm{~S}\right) \otimes \mathrm{L}^{2}\left(\mathrm{~S}^{2 \mathrm{k}}, \operatorname{Cliff}_{\theta}\left(\mathrm{S}^{2 \mathrm{k}}\right)\right)\right]^{\mathrm{SO}(2 \mathrm{k})}
$$

As is shown in [10, Section 3], there is a first-order, formally self-adjoint, $\mathrm{SO}(2 \mathrm{k})$ equivariant operator $\mathrm{D}$ acting on the sections of $\pi^{*} S$ over $\mathrm{P}$ such that the same formula we have been using,

$$
\widehat{\mathrm{D}}=\mathrm{D} \otimes \varepsilon+\mathrm{I} \otimes \mathrm{D}_{\theta}
$$


defines a Dirac operator for $(\widehat{M}, \hat{S}, \hat{f})$. If we define J and P exactly as above, then we can repeat the argument given in the case where $\mathrm{P}$ is trivial, noting in addition that the subspace

$$
\mathrm{L}^{2}\left(\mathrm{P}, \pi^{*} S\right)^{\mathrm{SO}(2 \mathrm{k})} \otimes \operatorname{ker}\left(\mathrm{D}_{\theta}\right) \subseteq\left[\mathrm{L}^{2}\left(\mathrm{P}, \pi^{*} \mathrm{~S}\right) \otimes \mathrm{L}^{2}\left(\mathrm{~S}^{2 \mathrm{k}}, \mathrm{Cliff}_{\theta}\left(\mathrm{S}^{2 \mathrm{k}}\right)\right)\right]^{\mathrm{SO}(2 \mathrm{k})}
$$

(this is the range of $\mathrm{P}^{\perp}$ ) identifies with $\mathrm{L}^{2}(\mathrm{M}, \mathrm{S})$, and the restriction of $\mathrm{D} \otimes \varepsilon$ to this subspace defines a Dirac operator D for $(M, S, f)$.

Let us reformulate Theorem 6.1 as follows:

6.8. Theorem. The correspondence that associates to each geometric K-cycle $(\mathrm{M}, \mathrm{S}, \mathrm{f})$ for $\mathrm{B} \pi$ the relative eta invariant, modulo $\mathbb{Z}$, of a Dirac operator for $(M, S, f)$ determines a group homomorphism $\operatorname{Ind}_{\sigma_{1}, \sigma_{2}}: \mathrm{K}_{1}(\mathrm{X}) \longrightarrow \mathbb{R} / \mathbb{Z}$.

Atiyah Patodi and Singer consider essentially the same $\mathbb{R} / \mathbb{Z}$-index map in [5], except that they work with the K-theory of TX rather than the K-homology of $X$ (they deal only with manifolds). The K-homology point of view has some conceptual advantages, since built into it are invariance properties (for instance, bordism invariance) of the $\mathbb{R} / \mathbb{Z}$-index. Here are two applications. The results, with different proofs, are due to Weinberger [16].

6.9. Theorem. Let $M$ be a closed, odd-dimensional spin-manifold with positive scalar curvature, let $\mathrm{D}$ be the Dirac operator on $\mathrm{M}$, and let $\mathrm{f}: \mathrm{M} \rightarrow \mathrm{B} \pi$ be a continuous map. For any pair of representations $\sigma_{1}, \sigma_{2}: \pi \rightarrow \mathrm{U}(\mathrm{N})$, the relative eta invariant $\rho\left(\mathrm{D}, \mathrm{f}, \sigma_{1}, \sigma_{2}\right)$ is a rational number.

Proof. Let $\pi_{0}$ be the direct product of the two images of $\pi$ in $U(N)$ under $\sigma_{1}$ and $\sigma_{2}$. Then $\pi_{0}$ is a linear group and there is a group homomorphism $\mathrm{k}: \pi \rightarrow \pi_{0}$ through which both $\sigma_{1}$ and $\sigma_{2}$ factor:

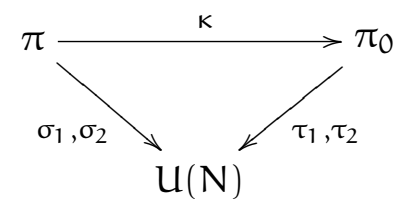

The group homomorphism $\kappa: \pi \rightarrow \pi_{0}$ determines a map of classifiying spaces $\mathrm{k}: \mathrm{B} \pi \longrightarrow \mathrm{B} \pi_{0}$, and

$$
\rho\left(D, f, \sigma_{1}, \sigma_{2}\right)=\rho\left(D, k \circ f, \tau_{1}, \tau_{2}\right) .
$$


Now it is proved in [18] that for $\pi_{0}$ (or any linear group) the assembly map

$$
\mu: \mathrm{K}_{*}\left(\mathrm{~B} \pi_{0}\right) \rightarrow \mathrm{K}_{*}\left(\mathrm{C}^{*}\left(\pi_{0}\right)\right)
$$

is rationally injective. But if $M$ is an odd-dimensional, positive scalar curvature spin manifold, and if $S$ is its spinor bundle, then for any map $g: M \rightarrow B \pi_{0}$, the K-cycle $(M, S, g)$ determines an element of the kernel of the assembly map (see Section 7$)$ and hence is a torsion element of $K_{1}\left(B \pi_{0}\right)$. So the relative eta invariant of the Dirac operator is a torsion element of $\mathbb{R} / \mathbb{Z}$. In other words it is a rational number.

6.10. Remark. The same argument shows that if the assembly map for $\pi$ itself is injective (for example, if $\pi$ is linear and torsion-free), then the relative eta invariant $\rho\left(D, f, \sigma_{1}, \sigma_{2}\right)$ is an integer.

6.11. Theorem. Let $\mathrm{h}: \mathrm{M}^{\prime \prime} \rightarrow \mathrm{M}^{\prime}$ be an orientation-preserving homotopy equivalence between odd-dimensional, closed, oriented manifolds. Let $\mathrm{f}^{\prime}: \mathrm{M}^{\prime} \rightarrow \mathrm{B} \pi$ be a continuous map, and let $\mathrm{f}^{\prime \prime}=\mathrm{f}^{\prime} \circ \mathrm{h}: \mathrm{M}^{\prime \prime} \rightarrow \mathrm{B} \pi$. Let $\mathrm{D}_{\mathrm{M}^{\prime}}$ and $\mathrm{D}_{\mathrm{M}^{\prime \prime}}$ be the signature operators on $M^{\prime}$ and $M^{\prime \prime}$. For any pair of representations $\sigma_{1}, \sigma_{2}: \pi \rightarrow U(N)$, the relative eta invariants

$$
\rho\left(D_{M^{\prime}}, f^{\prime}, \sigma_{1}, \sigma_{2}\right) \quad \text { and } \rho\left(D_{M^{\prime \prime}}, f^{\prime \prime}, \sigma_{1}, \sigma_{2}\right)
$$

are equal to one another, modulo rational numbers.

Proof. The argument is the same. The signature operators individually determine elements in K-homology from which their relative eta invariants may be recovered, modulo $\mathbb{Z}$, and as we shall review in Section 7 , the difference of these elements maps to zero under the assembly map.

6.12. Remark. The definition of the $\mathbb{R} / \mathbb{Z}$-index map on K-homology has other uses too. For example it should allow one to conceptualize the Atiyah-PatodiSinger $\mathbb{R} / \mathbb{Z}$-index theorem, very much as Baum's geometric definition of Khomology allows one to conceptualize the original Atiyah-Singer index theorem. But we shall not pursue this here.

\section{Structures}

In this section we shall define structure invariants in $\mathcal{S}_{1}(\pi)$ associated to positive scalar curvature metrics on spin manifolds and to homotopy equivalences between oriented manifolds. 
The invariant associated to an odd-dimensional, closed spin manifold with postive scalar curvature is easy to descibe. Let $\mathrm{D}$ be the spinor Dirac operator. The Lichnerowicz formula applies equally to $\mathrm{D}$ or to its lift $\mathrm{D}_{\pi}$ to the Mishchenko line bundle L, and so

$$
\mathrm{D}_{\pi}^{2}=\nabla_{\pi}^{*} \nabla_{\pi}+\frac{\mathrm{k}}{4}
$$

where $\mathrm{K}$ is the scalar curvature function and $\nabla_{\pi}$ is the spin-connection operator on $\mathrm{L} \otimes \mathrm{S}$. It follows that if the scalar curvature is bounded below by $k^{2}$, then the spectrum of $D_{\pi}$ is disjoint from the interval $\left(-\frac{k}{2}, \frac{k}{2}\right)$. We may therefore form the involution $\operatorname{sign}\left(D_{\pi}\right) \in D_{\pi}^{*}(M)$ and the projection

$$
\mathrm{P}_{\pi}=\frac{1}{2}\left(\operatorname{sign}\left(\mathrm{D}_{\pi}\right)+\mathrm{I}\right)
$$

using the continuous functional calculus.

7.1. Definition. Let $M$ be a closed, odd-dimensional spin manifold with positive scalar curvature, and let $f: M \rightarrow B \pi$ be any map. Denote by $[M, f] \in \mathcal{S}_{1}(\pi)$ the class determined by the above projection $\mathrm{P}_{\pi}$.

In order to define the structure invariant associated to a homotopy equivalence between closed, oriented manifolds we need the following K-theory construction.

7.2. Definition. Let $A$ be a unital $C^{*}$-algebra and let $J$ be a closed $C^{*}$-algebra ideal in $A$. Denote by $\check{K}_{0}(A)$ the Grothendieck group of homotopy classes of triples $(\gamma, \tilde{p}, p)$ where:

(a) $p$ is a projection in $M_{\infty}(A / J)$.

(b) $\tilde{p}$ is a lift of $p$ to an element of $M_{\infty}(A)$.

(c) $\gamma:[0,2] \rightarrow \mathrm{GL}_{\infty}(\mathrm{J})$ is a path with $\gamma(0)=\mathrm{I}$ and $\gamma(2)=\exp (2 \pi i \tilde{p})$.

The addition operation on homotopy classes of triples is given by direct sum.

7.3. Remark. We recall that $\mathrm{GL}_{\mathfrak{n}}(J)$ is the topological group of matrices in $\mathrm{GL}_{n}(A)$ that are equal to the identity, modulo $M_{n}(J)$ and that $G L_{\infty}(J)$ is the inductive limit of the $\mathrm{GL}_{n}(\mathrm{~J})$ under the standard embeddings. We give it the inductive limit topology, under which any path $\gamma$, as in item (c), automatically lies in some $\mathrm{GL}_{n}(\mathrm{~J})$.

The notation $\check{K}_{0}(A)$ has the shortcoming of not making any reference to the ideal J. But the omission is somewhat justified by of the following calculation: 
7.4. Lemma. The map from $\mathrm{K}_{0}(A)$ into $\check{\mathrm{K}}_{0}(\mathrm{~A})$ that sends the class of a projection $\mathrm{q} \in \mathrm{M}_{\mathrm{n}}(A)$ to the class of the triple $(\gamma, \mathrm{q}, \dot{\mathrm{q}})$, where

(a) $\dot{\mathrm{q}}$ is the image of $\mathrm{q}$ in $\mathrm{M}_{\infty}(\mathrm{A} / \mathrm{J})$, and

(b) $\gamma(\mathrm{t}) \equiv \mathrm{I}$

is an isomorphism of abelian groups.

Proof. Denote by $\mathrm{K}_{2}(A)$ the fundamental group of $\mathrm{GL}_{\infty}(A)$ and define a map from $\check{K}_{0}(A)$ into $K_{2}(A)$ by sending a triple $(\gamma, \tilde{p}, p)$ to the homotopy class of the loop

$$
t \mapsto \begin{cases}\gamma(2 t) & 0 \leq t \leq 1 \\ \exp (2 \pi i(2-t) \tilde{p}) & 1 \leq t \leq 2\end{cases}
$$

The composition

$$
\mathrm{K}_{0}(\mathrm{~A}) \longrightarrow \check{\mathrm{K}}_{0}(\mathrm{~A}) \longrightarrow \mathrm{K}_{2}(\mathrm{~A})
$$

is the standard Bott periodicity isomorphism, and this shows that the map in the statement of the lemma is a (split) injection. To prove surjectivity, consider the diagram

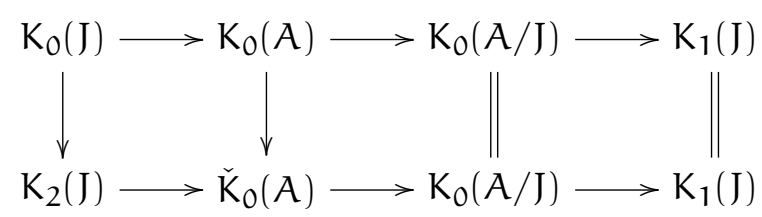

in which:

(a) the first vertical map is the Bott periodicity isomorphsm and the second is the one given in the statement of the lemma;

(b) the map of $K_{2}(J)$ into $\check{K}_{0}(A)$ sends the class of a loop $\gamma$ to the class of the triple $(\gamma, 0,0)$;

(c) the map of $\check{K}_{0}(A)$ into $K_{0}(A / J)$ sends the class of a triple $(\gamma, \tilde{p}, p)$ into the class of $p$; and

(d) the remaining maps are the standard ones in the K-theory exact sequence.

The rows are exact, and so a diagram chase completes the proof.

Applying this calculation to the K-theory groups that are used to define the structure group $\mathcal{S}_{1}(\pi)$, we conclude from Definition 7.2 and Lemma 7.4 that an element of the structure group $S_{1}(\pi)$ is determined by the following data: 
(a) a projection $P$ in $Q_{H}^{*}(X)$, where $X$ is a finite subcomplex of $B \pi$ and $H$ is a Hilbert space equipped with a representation of $C(X)$, and

(b) a lift $\tilde{\mathrm{P}}$ to $\mathrm{D}_{\mathrm{H}_{\pi}}^{*}(\mathrm{X})$ and a path from the identity on $\mathrm{H}_{\pi}$ to $\exp (2 \pi i \tilde{\mathrm{P}})$ through invertible operators that are compact perturbations of the identity operator.

7.5. Example. Consider the analytic K-cycle for $\mathrm{X} \subseteq \mathrm{B} \pi$ determined by a geometric K-cycle $(M, S, f)$ with $f[M] \subseteq X$. If $D$ is a Dirac operator associated to $(M, S, f)$, then we noted in Example 5.12 that we may form the operator

$$
\tilde{\mathrm{P}}_{\pi}=\frac{1}{2}\left(\frac{2}{\pi} \arctan \left(\mathrm{D}_{\pi}\right)+\mathrm{I}\right) \in \mathrm{D}_{\mathrm{H}_{\pi}}^{*}(\mathrm{X}) \text {, }
$$

which lifts the projection $\mathrm{P}=\frac{1}{2}(\operatorname{sign}(\mathrm{D})+\mathrm{I}) \in \mathrm{Q}_{\mathrm{H}}^{*}(\mathrm{X})$ that determines the analytic K-homology class of $(M, S, f)$. Moreover, we noted that

$$
\exp (2 \pi i \tilde{P})=\left(D_{\pi}-i I\right)\left(D_{\pi}+i I\right)^{-1} .
$$

So to associate to $(M, S, f)$ a structure in $\mathcal{S}_{1}(\pi)$ (should one exist) it suffices to choose an appropriate Dirac operator D, and then a continuous path $\gamma_{\pi}$ of invertible operators, all of them compact perturbations of the identity, connecting the Cayley transform of $\mathrm{D}_{\pi}$ to the identity.

Now let $h: M^{\prime \prime} \rightarrow M^{\prime}$ be an orientation-preserving smooth homotopy equivalence between oriented, smooth closed manifolds. Let $f^{\prime}: M^{\prime} \rightarrow B \pi$ be any map and let $f^{\prime \prime}=f^{\prime} \circ h$. Consider the geometric K-cycle

$$
\left(M^{\prime}, S^{\prime}, f^{\prime}\right) \sqcup-\left(M^{\prime \prime}, S^{\prime \prime}, f^{\prime \prime}\right),
$$

where $S^{\prime}$ and $S^{\prime \prime}$ are the even-degree parts of the exterior algebra bundles of $M^{\prime}$ and $M^{\prime \prime}$. They are given the Dirac bundle structures for which the signature operators $\pm \mathfrak{i}(\mathrm{d} *-* \mathrm{~d})$ are Dirac operators (the signs will be specified below). We shall describe an explicit path $\gamma_{\pi}$ connecting the Cayley transform of the direct sum of $\mathrm{D}_{\pi}^{\prime}$ and $-\mathrm{D}_{\pi}^{\prime \prime}$ to the identity.

The construction follows [23, Section 5], to which we refer the reader for details. Let $M$ be either of $M^{\prime}$ of $M^{\prime \prime}$ and write $\operatorname{dim}(M)=2 \ell+1$. Define a self-adjoint involution $\mathrm{J}$ of the bundle $\mathrm{S}$ using the Hodge $*$-operator and the formula

$$
\mathrm{J} \omega=i^{\mathrm{p}(\mathrm{p}-1)+\ell} * \omega
$$

on $p$-forms. The operator $J$ anticommutes with the de Rham operator $B=d+d^{*}$, and the signature operator is by definition

$$
\mathrm{D}=\mathrm{iBJ} .
$$


We see therefore that

$$
\begin{aligned}
(\mathrm{D}-\mathrm{iI})(\mathrm{D}+\mathrm{iI})^{-1}=(\mathrm{iBJ}- & \mathrm{iI})(i \mathrm{BJ}+\mathrm{iI})^{-1} \\
& =(\mathrm{B}-\mathrm{J})(\mathrm{iJ})(\mathrm{iJ})^{-1}(\mathrm{~B}+\mathrm{J})^{-1}=(\mathrm{B}-\mathrm{J})(\mathrm{B}+\mathrm{J})^{-1} .
\end{aligned}
$$

We need to connect the direct sum of the Cayley transforms of $D_{\pi}^{\prime}$ and $-D_{\pi}^{\prime \prime}$ to the identity, and in view of the above calculation this amounts to connecting the operator $\left(\mathbb{B}_{\pi}-\mathbb{J}_{\pi}\right)\left(\mathbb{B}_{\pi}+\mathbb{J}_{\pi}\right)^{-1}$ to the identity, where

$$
\mathbb{B}_{\pi}=\left(\begin{array}{cc}
\mathrm{B}_{\pi}^{\prime} & 0 \\
0 & \mathrm{~B}_{\pi}^{\prime \prime}
\end{array}\right) \quad \text { and } \quad \mathbb{J}_{\pi}=\left(\begin{array}{cc}
\mathrm{J}_{\pi}^{\prime} & 0 \\
0 & -\mathrm{J}_{\pi}^{\prime \prime}
\end{array}\right)
$$

The major part of the path takes the form $\left(\mathbb{B}_{\pi}-\mathbb{J}_{\pi, t}\right)\left(\mathbb{B}_{\pi}+\mathbb{J}_{\pi, t}\right)^{-1}$ and involves the homotopy equivalence $h: M^{\prime \prime} \rightarrow M^{\prime}$ as follows. Let $\Delta_{\pi}^{\prime}$ and $\Delta_{\pi}^{\prime \prime}$ be the Laplace operators on forms (with coefficients in the Mishchenko line bundle) and define a bounded (in fact compact) operator

$$
A_{\pi}=\exp \left(-\Delta_{\pi}\right) h^{*} \exp \left(-\Delta_{\pi}^{\prime \prime}\right): \mathrm{L}_{\pi}^{2}\left(M^{\prime \prime}, S^{\prime \prime}\right) \longrightarrow \mathrm{L}_{\pi}^{2}\left(M^{\prime}, S^{\prime}\right)
$$

by applying $\exp \left(-\Delta_{\pi}^{\prime \prime}\right)$ to an $\mathrm{L}^{2}$-form on $\mathrm{M}^{\prime \prime}$ so as to obtain, by elliptic regularity, a smooth form, and then pulling back this smooth form to $M^{\prime}$ using the smooth map $h$ (and then applying $\exp \left(-\Delta_{\pi}^{\prime}\right)$ for good measure - this last step is unnecessary here, but it will be used in Section 9). The operator $A_{\pi}$ induces an isomorphism on de Rham cohomology groups and it follows from [23, Section 5] that if

$$
\mathbb{J}_{\pi, \mathrm{t}}=\left(\begin{array}{cc}
J_{\pi}^{\prime} & 0 \\
0-2 \mathrm{t} A_{\pi} J_{\pi}^{\prime} A_{\pi}^{*}+(2 t-1) J_{\pi}^{\prime \prime}
\end{array}\right) \quad\left(0 \leq t \leq \frac{1}{2}\right)
$$

and

$$
\mathbb{J}_{\pi, t}=\left(\begin{array}{cc}
\sin (\pi \mathrm{t}) J_{\pi}^{\prime} & \cos (\pi \mathrm{t}) J_{\pi}^{\prime} A_{\pi}^{*} \\
\cos (\pi \mathrm{t}) A_{\pi} J_{\pi}^{\prime} & -\sin (\pi \mathrm{t}) A_{\pi} J_{\pi}^{\prime} A_{\pi}^{*}
\end{array}\right) \quad\left(\frac{1}{2} \leq \mathrm{t} \leq 1\right),
$$

then all the operators $\left(\mathbb{B}_{\pi}-\mathbb{J}_{\pi, \mathrm{t}}\right)\left(\mathbb{B}_{\pi}+\mathbb{J}_{\pi, \mathrm{t}}\right)^{-1}$ are well-defined (that is, the inverses exist) and form a continuous path of invertible operators, each a compact perturbation of the identity. We obtain a path from $\left(\mathbb{B}_{\pi}-\mathbb{J}_{\pi}\right)\left(\mathbb{B}_{\pi}+\mathbb{J}_{\pi}\right)^{-1}$ to $\left(\mathbb{B}_{\pi}-\mathbb{J}_{\pi, 1}\right)\left(\mathbb{B}_{\pi}+\mathbb{J}_{\pi, 1}\right)^{-1}$. If we define

$$
\mathbb{J}_{\pi, t}=\left(\begin{array}{cc}
0 & e^{\pi i t} J_{\pi}^{\prime} A_{\pi}^{*} \\
e^{-\pi i t} A_{\pi} J_{\pi}^{\prime} & 0
\end{array}\right) \quad(1 \leq t \leq 2)
$$


then the path of operators $\left(\mathbb{B}_{\pi}-\mathbb{J}_{\pi, 1}\right)\left(\mathbb{B}_{\pi}+\mathbb{J}_{\pi, t}\right)^{-1}$ is well-defined also, and since $\mathbb{J}_{\pi, 1}=-\mathbb{J}_{\pi, 2}$ ends at the identity. The concatenation of this with the previous path connects $(\mathbb{B}-\mathbb{J})(\mathbb{B}+\mathbb{J})^{-1}$ to the identity, as required.

7.6. Definition. Let $h: M^{\prime \prime} \rightarrow M^{\prime}$ be an orientation-preserving homotopy equivalence between odd-dimensional, closed, oriented manifolds. Let $f^{\prime}: M^{\prime} \rightarrow B \pi$ be any map, and let $f^{\prime \prime}=f^{\prime} \circ h: M^{\prime \prime} \rightarrow B \pi$. Denote by $\left[h, f^{\prime}, f^{\prime \prime}\right] \in \mathcal{S}_{1}(\pi)$ the class determined by the disjoint union

$$
\left(M^{\prime}, S^{\prime}, f^{\prime}\right) \sqcup-\left(M^{\prime \prime}, S^{\prime \prime}, f^{\prime \prime}\right),
$$

where $S^{\prime}$ and $S^{\prime \prime}$ are the Dirac bundle determining the signature operators $D^{\prime}$ and $D^{\prime \prime}$ on $M^{\prime}$ and $M^{\prime \prime}$, together with the path $\gamma_{\pi}$ described above connecting the Cayley transform of the direct sum of $\mathrm{D}_{\pi}^{\prime}$ and $-\mathrm{D}_{\pi}^{\prime \prime}$ to the identity.

\section{The Relative Trace}

In this section we shall define the relative trace $\operatorname{Tr}_{\sigma_{1}, \sigma_{2}}: \mathcal{S}_{1}(\pi) \rightarrow \mathbb{R}$, discussed in the introduction, which fits into a commuting diagram

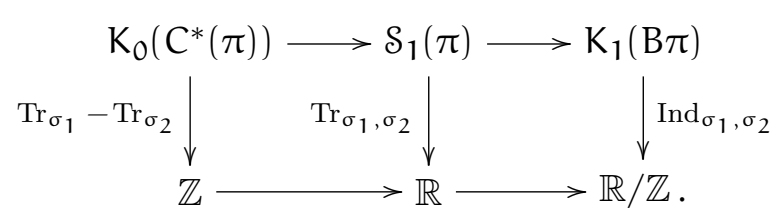

We shall do so by introducing a version of the structure group $\mathcal{S}_{1}(\pi)$, denoted $\mathcal{S}_{2}\left(\sigma_{1}, \sigma_{2}\right)$, that is specifically tailored to the pair of representations $\sigma_{1}, \sigma_{2}: \pi \rightarrow$ $\mathrm{U}(\mathrm{N})$. There will be a natural map from $\mathcal{S}_{1}(\pi)$ into $\mathcal{S}_{1}\left(\sigma_{1}, \sigma_{2}\right)$. We shall also define a geometric group $\mathcal{S}_{1}^{\text {geom }}\left(\sigma_{1}, \sigma_{2}\right)$ using ideas borrowed from geometric $K$ homology theory, and then define a functional $\rho: \mathcal{S}_{1}^{\text {geom }}\left(\sigma_{1}, \sigma_{2}\right) \rightarrow \mathbb{R}$ using relative eta invariants (compare Section 6). Finally, we shall construct an isomorphism

$$
\mathcal{S}_{1}^{\text {geom }}\left(\sigma_{1}, \sigma_{2}\right) \underset{\mathcal{S}_{1}}{\cong}\left(\sigma_{1}, \sigma_{2}\right)
$$

and obtain the relative trace from the diagram

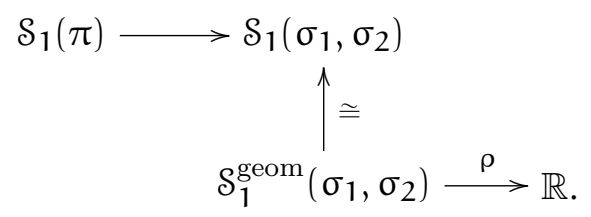


Let $X$ be a compact space and let $f: X \rightarrow B \pi$ be any map. Let $H$ be a separable Hilbert space that is equipped with a representation of $C(X)$. Denote by $V_{1}$ and $V_{2}$ the flat Hermitian vector bundles over $X$ associated to the representations $\sigma_{1}$ and $\sigma_{2}$ and the map $f$, and form the Hilbert spaces

$$
\mathrm{H}_{1}=\Gamma\left(\mathrm{V}_{1}\right) \otimes_{\mathrm{C}(\mathrm{X})} \mathrm{H} \quad \text { and } \quad \mathrm{H}_{2}=\Gamma\left(\mathrm{V}_{2}\right) \otimes_{\mathrm{C}(\mathrm{X})} \mathrm{H} .
$$

Both $\mathrm{H}_{1}$ and $\mathrm{H}_{2}$ carry representations of $\mathrm{C}(\mathrm{X})$ associated to the natural left action of $\mathrm{C}(\mathrm{X})$ on the modules $\Gamma\left(\mathrm{V}_{1}\right)$ and $\Gamma\left(\mathrm{V}_{2}\right)$. In the following definition we shall use the notion of a lift of an operator in $\mathrm{D}_{\mathrm{H}}^{*}(\mathrm{X})$ that was introduced in Definition 5.3.

8.1. Definition. Let $\mathrm{D}_{\mathrm{H}_{1}, \mathrm{H}_{2}}^{*}(\mathrm{X})$ be the $\mathrm{C}^{*}$-algebra

$$
\mathrm{D}_{\mathrm{H}_{1}, \mathrm{H}_{2}}^{*}(\mathrm{X})=\left\{\left(\mathrm{T}_{1}, \mathrm{~T}_{2}\right) \in \mathrm{D}_{\mathrm{H}_{1}}(\mathrm{X}) \oplus \mathrm{D}_{\mathrm{H}_{2}}(\mathrm{X}): \begin{array}{c}
\mathrm{T}_{1} \text { and } \mathrm{T}_{2} \text { lift some } \\
\text { common } \mathrm{T} \in \mathrm{D}_{\mathrm{H}}^{*}(\mathrm{X})
\end{array}\right\} .
$$

8.2. Remark. The map that associates to an element $\left(T_{1}, T_{2}\right) \in D_{H_{1}, H_{2}}^{*}(X)$ the class in $Q_{H}^{*}(X)$ of any operator $T$ that $T_{1}$ and $T_{2}$ both lift gives rise to a short exact sequence of $\mathrm{C}^{*}$-algebras

$$
0 \longrightarrow \mathcal{K}\left(\mathrm{H}_{1}\right) \oplus \mathcal{K}\left(\mathrm{H}_{2}\right) \longrightarrow \mathrm{D}_{\mathrm{H}_{1}, \mathrm{H}_{2}}^{*}(\mathrm{X}) \longrightarrow \mathrm{Q}_{\mathrm{H}}^{*}(\mathrm{X}) \longrightarrow 0
$$

(recall that $\mathrm{Q}_{\mathrm{H}}^{*}(\mathrm{X})$ is the quotient of $\mathrm{D}_{\mathrm{H}}^{*}(\mathrm{X})$ by the compact operators).

8.3. Definition. Let $\mathrm{H}$ be a separable Hilbert space equipped with an ample representation of $\mathrm{C}(\mathrm{X})$. Denote by $\mathcal{S}_{1}\left(\sigma_{1}, \sigma_{2}, \mathrm{X}\right)$ the $\mathrm{K}$-theory group

$$
\mathcal{S}_{*}\left(\sigma_{1}, \sigma_{2}, X\right)=\mathrm{K}_{0}\left(D_{\mathrm{H}_{1}, \mathrm{H}_{2}}^{*}(X)\right) .
$$

This is independent of the choice of $\mathrm{H}$, up to canonical isomorphism. The group $\mathcal{S}_{1}\left(\sigma_{1}, \sigma_{2}, X\right)$ is covariantly functorial in $X$ on the category of compact metrizable spaces $X$ that are equipped with maps to $B \pi$, and we can therefore define the structure group $\mathcal{S}_{1}\left(\sigma_{1}, \sigma_{2}\right)$ as follows:

8.4. Definition. We shall write

$$
\mathcal{S}_{1}\left(\sigma_{1}, \sigma_{2}\right)=\underset{X \subseteq \mathrm{B} \pi}{\lim _{\mathrm{B}}} \mathcal{S}_{1}\left(\sigma_{1}, \sigma_{2}, \mathrm{X}\right),
$$

where the direct limit is over finite subcomplexes of $B \pi$.

The Hilbert spaces $\mathrm{H}_{1}$ and $\mathrm{H}_{2}$ can be described as tensor products

$$
\mathrm{H}_{1}=\mathrm{H}_{\pi} \otimes_{\mathrm{C}^{*}(\pi)} \mathbb{C}_{\sigma_{1}}^{\mathrm{N}} \text { and } \mathrm{H}_{2}=\mathrm{H}_{\pi} \otimes_{\mathrm{C}^{*}(\pi)} \mathbb{C}_{\sigma_{2}}^{\mathrm{N}} \text {, }
$$


where $\mathbb{C}_{\sigma_{1}}^{N}$ and $\mathbb{C}_{\sigma_{2}}^{N}$ denote copies of the Hilbert space $\mathbb{C}^{N}$ that are equipped with the left actions of $C^{*}(\pi)$ determined by the representations $\sigma_{1}$ and $\sigma_{2}$. Because of this, the formula

$$
\mathrm{T}_{\pi} \mapsto\left(\mathrm{T}_{\pi} \otimes \mathrm{I}, \mathrm{T}_{\pi} \otimes \mathrm{I}\right)
$$

gives a natural homomorphism of $\mathrm{C}^{*}$-algebras from $\mathrm{D}_{\mathrm{H}_{\pi}}^{*}(\mathrm{X})$ into $\mathrm{D}_{\mathrm{H}_{1}, \mathrm{H}_{2}}^{*}(\mathrm{X})$, and hence a natural homomorphism of abelian groups

$$
\mathcal{S}_{1}(\pi) \longrightarrow \mathcal{S}_{1}\left(\sigma_{1}, \sigma_{2}\right) .
$$

Before defining the geometric group $\mathcal{S}_{1}^{\text {geom }}\left(\sigma_{1}, \sigma_{2}\right)$, let us analyze further the relationship between $\mathcal{S}_{1}\left(\sigma_{1}, \sigma_{2}\right)$ and $\mathcal{S}_{1}(\pi)$ using the following calculation:

8.5. Lemma. If $\mathrm{H}$ is equipped with an ample representation of $\mathrm{C}(\mathrm{X})$, then the image of the connecting homomorphism

$$
\mathrm{K}_{1}\left(\mathrm{Q}_{\mathrm{H}}^{*}(\mathrm{X})\right) \longrightarrow \mathrm{K}_{0}\left(\mathcal{K}\left(\mathrm{H}_{1}\right) \oplus \mathcal{K}\left(\mathrm{H}_{2}\right)\right)
$$

associated to the short exact sequence in Remark 8.2 is the diagonal copy of $\mathbb{Z}$ in the group $\mathrm{K}_{0}\left(\mathcal{K}\left(\mathrm{H}_{1}\right) \oplus \mathcal{K}\left(\mathrm{H}_{2}\right)\right) \cong \mathbb{Z} \oplus \mathbb{Z}$.

Proof. Since the bundles $\mathrm{V}_{1}$ and $\mathrm{V}_{2}$ used to define $\mathrm{H}_{1}$ and $\mathrm{H}_{2}$ are flat, there is a positive integer $k$ such that the $k$-fold direct sums

$$
\mathrm{V}_{1}^{\mathrm{k}}=\mathrm{V}_{1} \oplus \cdots \oplus \mathrm{V}_{1} \quad \text { and } \quad \mathrm{V}_{2}^{\mathrm{k}}=\mathrm{V}_{2} \oplus \cdots \oplus \mathrm{V}_{2}
$$

are both trivializable as smooth vector bundles. Since

$$
\Gamma\left(\mathrm{V}_{1}\right) \otimes_{\mathrm{C}(\mathrm{X})} \mathrm{H}^{\mathrm{k}}=\Gamma\left(\mathrm{V}_{1}^{\mathrm{k}}\right) \otimes_{\mathrm{C}(\mathrm{X})} \mathrm{H} \quad \text { and } \quad \Gamma\left(\mathrm{V}_{2}\right) \otimes_{\mathrm{C}(\mathrm{X})} \mathrm{H}^{\mathrm{k}}=\Gamma\left(\mathrm{V}_{2}^{\mathrm{k}}\right) \otimes_{\mathrm{C}(\mathrm{X})} \mathrm{H},
$$

it follows from the stability of K-theory that the K-theory sequence we are considering identifies with the one associated to the short exact sequence

$$
0 \longrightarrow \mathcal{K}\left(\mathrm{H}^{\mathrm{kN}}\right) \oplus \mathcal{K}\left(\mathrm{H}^{\mathrm{kN}}\right) \longrightarrow \mathrm{D}_{\mathrm{H}^{\mathrm{kN}}, \mathrm{H}^{\mathrm{kN}}}^{*}(\mathrm{X}) \longrightarrow \mathrm{Q}_{\mathrm{H}^{\mathrm{kN}}}^{*}(\mathrm{X}) \longrightarrow 0,
$$

where

$$
\mathrm{D}_{\mathrm{H}^{k N}, H^{k N}}^{*}(X)=\left\{\left(\mathrm{T}_{1}, \mathrm{~T}_{2}\right) \in \mathrm{D}_{\mathrm{H}^{k N}}(\mathrm{X}) \oplus \mathrm{D}_{\mathrm{H}^{\mathrm{kN}}}(\mathrm{X}): \mathrm{T}_{1}-\mathrm{T}_{2} \in \mathcal{K}(\mathrm{H})\right\} .
$$

The lemma follows from this.

8.6. Definition. If $n \in \mathbb{Z}$, then denote by $[n] \in \mathcal{S}_{1}\left(\sigma_{1}, \sigma_{2}\right)$ the $K$-theory class of any projection $\left(e_{1}, e_{2}\right) \in \mathrm{D}_{\mathrm{H}_{1}, \mathrm{H}_{2}}^{*}(X)$, where $e_{1}$ and $e_{2}$ have finite rank, and

$$
\operatorname{rank}\left(e_{1}\right)-\operatorname{rank}\left(e_{2}\right)=n
$$


(by the lemma, all these projections have the same K-theory class).

Because of Lemma 8.5 and the vanishing of $K_{1}$ for the compact operators, the K-theory sequence in Lemma 8.5 gives rise (after passing to a direct limit over the finite subcomplexes $\mathrm{X} \subseteq \mathrm{B} \pi$ ) to a short exact sequence

$$
0 \longrightarrow \mathbb{Z} \longrightarrow \mathcal{S}_{1}\left(\sigma_{1}, \sigma_{2}\right) \longrightarrow \mathrm{K}_{1}(\mathrm{~B} \pi) \longrightarrow 0,
$$

in which the integer $n \in \mathbb{Z}$ is sent to the class $[n] \in \mathcal{S}_{1}\left(\sigma_{1}, \sigma_{2}\right)$. We obtain a commuting diagram

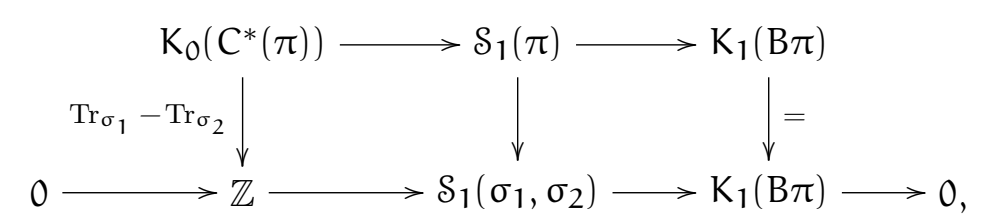

in which the map from $\mathrm{K}_{0}\left(\mathrm{C}^{*}(\pi)\right)$ to $\mathbb{Z}$ assigns to a projection matrix $p$ over $C^{*}(\pi)$ the difference of the traces of $p$ in the representations $\sigma_{1}$ and $\sigma_{2}$.

We turn now to the definition of the geometric structure group $\mathcal{S}_{1}\left(\sigma_{1}, \sigma_{2}\right)$.

8.7. Definition. An (odd) geometric $\left(\sigma_{1}, \sigma_{2}\right)$-cycle is a quintuple $(M, S, f, D, n)$ where

(a) $(M, S, f)$ is a geometric K-cycle for $B \pi$.

(b) $\mathrm{D}$ is a specific choice of Dirac operator for $(M, S, f)$.

(c) $\mathrm{n}$ is an integer.

8.8. Remark. As it stands the definition of geometric cycle does not involve the representations $\sigma_{1}$ and $\sigma_{2}$. However the equivalence relation on cycles that determines the geometric structure group $\mathcal{S}_{1}\left(\sigma_{1}, \sigma_{2}\right)$ will depend on $\sigma_{1}$ and $\sigma_{2}$.

8.9. Definition. A geometric $\left(\sigma_{1}, \sigma_{2}\right)$-cycle $(M, S, f, D, n)$ is a boundary if there is a Dirac operator $\mathrm{Q}$ on a compact manifold $\mathrm{W}$ whose boundary is the operator $D$ on $M$, if the map $f$ extends to $W$, and if

$$
\operatorname{Ind}\left(\mathrm{Q}_{1}, \mathrm{D}_{1}\right)-\operatorname{Ind}\left(\mathrm{Q}_{2}, \mathrm{D}_{2}\right)=\mathrm{n} .
$$

Here, as usual, the subscripts 1 and 2 indicate twistings by the flat vector bundles $\mathrm{V}_{1}$ and $\mathrm{V}_{2}$.

8.10. Lemma. If a geometric $\left(\sigma_{1}, \sigma_{2}\right)$-cycle $(M, S, f, D, n)$ is a boundary, then

$$
\rho\left(D, f, \sigma_{1}, \sigma_{2}\right)+n=0 .
$$


Proof. This follows immediately from the formula in the proof of Lemma 2.4.

8.11. Definition. We shall call the quantity $\rho\left(D, f, \sigma_{1}, \sigma_{2}\right)+n$ the relative eta invariant of the geometric cycle $(M, S, f, D, n)$.

There is a natural notion of disjoint union of geometric $\left(\sigma_{1}, \sigma_{2}\right)$-cycles,

$$
\begin{aligned}
\left(M^{\prime}, S^{\prime}, f^{\prime}, D^{\prime}, n^{\prime}\right) \sqcup\left(M^{\prime \prime}, S^{\prime \prime}, f^{\prime \prime}, D^{\prime \prime}, n^{\prime \prime}\right) & \\
& =\left(M^{\prime} \sqcup M^{\prime \prime}, S^{\prime} \sqcup S^{\prime \prime}, f^{\prime} \sqcup f^{\prime \prime}, D^{\prime} \sqcup D^{\prime \prime}, n^{\prime}+n^{\prime \prime}\right) .
\end{aligned}
$$

There is also a notion of the negative of a cycle, but it involves a small subtlety. We should like the relative eta invariant of a cycle to be minus the relative eta invariant of the negative cycle. Since the relative eta invariant involves a correction term coming from the kernels of the twisted operators $D_{1}$ and $D_{2}$ we must therefore define

$$
-(M, S, f, D, n)=\left(M,-S, f,-D, d_{1}-d_{2}-n\right),
$$

where $d_{1}=\operatorname{dim} \operatorname{ker}\left(D_{1}\right)$ and $d_{2}=\operatorname{dim} \operatorname{ker}\left(D_{2}\right)$.

8.12. Definition. Two geometric $\left(\sigma_{1}, \sigma_{2}\right)$-cycles are bordant if the disjoint union of one with the negative of the other is a boundary.

We are now ready to define the geometric structure group:

8.13. Definition. We shall denote by $\mathcal{S}_{1}^{\text {geom }}\left(\sigma_{1}, \sigma_{2}\right)$ the set of equivalence classes of geometric $\left(\sigma_{1}, \sigma_{2}\right)$-cycles under the equivalence relation generated by:

(a) Direct sum/disjoint union. The cycle $\left(M, S^{\prime} \oplus S^{\prime \prime}, f, D^{\prime} \oplus D^{\prime \prime}, n\right)$ is equivalent to $\left(M \sqcup M, S^{\prime} \sqcup S^{\prime \prime}, f \sqcup f, D^{\prime} \oplus D^{\prime \prime}, n\right)$.

(b) Bordism. If $\left(M^{\prime}, S^{\prime}, f^{\prime}, D^{\prime}, n^{\prime}\right)$ and $\left(M^{\prime \prime}, S^{\prime \prime}, f^{\prime \prime}, D^{\prime \prime}, n^{\prime \prime}\right)$ are bordant, then they are equivalent.

(c) Bundle Modification. If $(\widehat{M}, \widehat{S}, \hat{f})$ is a bundle modification of $(M, S, f)$ in the sense of Definitions 3.9 and 3.10, and if

$$
\widehat{\mathrm{D}}=\mathrm{D} \otimes \varepsilon+\mathrm{I} \otimes \mathrm{D}_{\theta}
$$

is the specific Dirac operator that appears in the proof of Proposition 6.6, then $(M, S, f, D, n)$ and $(\hat{M}, \hat{S}, \hat{f}, \hat{D}, n)$ are equivalent.

As with geometric K-homology, the set $\mathcal{S}_{1}^{\text {geom }}\left(\sigma_{1}, \sigma_{2}\right)$ is an abelian group with addition given by disjoint union. 
The definition is designed with the following observation in mind:

8.14. Proposition. The relative eta invariant of a geometric $\left(\sigma_{1}, \sigma_{2}\right)$-cycle depends only on the class that the cycle determines in $\mathcal{S}_{1}\left(\sigma_{1}, \sigma_{2}\right)$.

Proof. It is clear that the direct sum/disjoint union relation preserves the relative eta invariant, and from the proof of Proposition 6.6 that the bundle modification relation does the same. Bordism is handled by Lemma 8.10.

8.15. Definition. We define the group homomorphism

$$
\rho: \mathcal{S}_{1}^{\text {geom }}\left(\sigma_{1}, \sigma_{2}\right) \longrightarrow \mathbb{R}
$$

by the formula

$$
\rho:(M, S, f, D, n) \mapsto \rho\left(D, f, \sigma_{1}, \sigma_{2}\right)+n .
$$

Our final task is to define an isomorphism from the geometric structure group $\mathcal{S}_{1}^{\text {geom }}\left(\sigma_{1}, \sigma_{2}\right)$ to the analytic group $\mathcal{S}_{1}\left(\sigma_{1}, \sigma_{2}\right)$.

8.16. Definition. If D is any Dirac operator on a closed manifold, then we shall denote by $p(D)$ the projection operator onto the direct sum of the eigenspaces of $\mathrm{D}$ associated with strictly positive eigenvalues.

Now let $(M, S, f, D, n)$ be a geometric $\left(\sigma_{1}, \sigma_{2}\right)$-cycle and let $X$ be any finite subcomplex of $B \pi$ that contains $f[M]$. View the Hilbert spaces $H_{1}$ and $H_{2}$ as equipped with representations of $C(X)$ via $f$, so that

$$
\left(p\left(D_{1}\right), p\left(D_{2}\right)\right) \in D_{\mathrm{H}_{1}, \mathrm{H}_{2}}^{*}(X) .
$$

8.17. Definition. Let $(M, S, f, D, n)$ be a geometric $\left(\sigma_{1}, \sigma_{2}\right)$-cycle. Its analytic structure class in the group $\mathcal{S}_{1}\left(\sigma_{1}, \sigma_{2}\right)$ is given by the formula

$$
\left[\left(p\left(D_{1}\right), p\left(D_{2}\right)\right]+[n] \in \mathcal{S}_{1}\left(\sigma_{1}, \sigma_{2}\right),\right.
$$

where $[n]$ is the class given in Definition 8.6.

We are going to prove the following result:

8.18. Theorem. The analytic structure class of a geometric cycle $(M, S, f, D, n)$ depends only on the class of $(\mathrm{M}, \mathrm{S}, \mathrm{f}, \mathrm{D}, \mathrm{n})$ in the geometric group $\mathcal{S}_{1}^{\text {geom }}\left(\sigma_{1}, \sigma_{2}\right)$. 
The key problem is to show that bordant cycles have the same analytic structure class. To solve it we shall use the work of Baum, Douglas and Taylor [9], who proved using boundary value theory that the analytic K-homology class determined by a geometric $\mathrm{K}$-cycle is unchanged under bordism.

8.19. Proposition. If the geometric $\left(\sigma_{1}, \sigma_{2}\right)$ cycle $(M, S, f, D, n)$ is a boundary, then its analytic structure class is equal to zero.

We shall divide the proof into several parts. First, let $W$ and $Q$ be a bounding manifold and operator, as in Definition 8.9. Let $\mathrm{Q}^{+}$be the component of $\mathrm{Q}$ that maps even-graded sections to odd-graded sections. We want to consider it as a Hilbert space operator with its maximal domain

$$
\operatorname{dom} \mathrm{Q}^{+}=\left\{\mathrm{s} \in \mathrm{L}^{2}\left(\mathrm{~W}, \mathrm{~S}^{+}\right): \mathrm{Q}^{+} \mathrm{s} \in \mathrm{L}^{2}\left(\mathrm{~W}, \mathrm{~S}^{-}\right)\right\} .
$$

We have written the $\mathbb{Z} / 2 \mathbb{Z}$-graded bundle on which $Q$ acts as $S^{+} \oplus S^{-}$. The operator $\mathrm{Q}^{+}$is closed, and we shall denote by $\mathrm{V}$ the partial isometry part of its polar decomposition.

Define $\mathrm{V}_{1}$ and $\mathrm{V}_{2}$ similarly, from the operators $\mathrm{Q}_{1}^{+}$and $\mathrm{Q}_{2}^{+}$obtained by twisting with the flat bundles associated to $\sigma_{1}$ and $\sigma_{2}$. We shall think of these as partial isometries on the Hilbert spaces $\mathrm{H}_{1}$ and $\mathrm{H}_{2}$ associated to the Hilbert space $\mathrm{H}=$ $\mathrm{L}^{2}\left(\mathrm{~W}, \mathrm{~S}^{+} \oplus \mathrm{S}^{-}\right)$.

8.20. Lemma. The pair $\left(\mathrm{V}_{1}, \mathrm{~V}_{2}\right)$ is an element of $\mathrm{D}_{\mathrm{H}_{1}, \mathrm{H}_{2}}^{*}(\mathrm{~W})$.

Proof. Consider the operator

$$
\mathrm{Q}=\left(\begin{array}{cc}
0 & \mathrm{Q}^{-} \\
\mathrm{Q}^{+} & 0
\end{array}\right): \mathrm{L}^{2}\left(\mathrm{~W}, \mathrm{~S}^{+} \oplus \mathrm{S}^{-}\right) \longrightarrow \mathrm{L}^{2}\left(\mathrm{~W}, \mathrm{~S}^{+} \oplus \mathrm{S}^{-}\right)
$$

where $\mathrm{Q}^{-}$is the adjoint of $\mathrm{Q}^{+}$. The partial isometry part of this operator in its polar decomposition is simply $\operatorname{sign}(\mathrm{Q})$ and is the $2 \times 2$ matrix assembled from the partial isometry parts of $\mathrm{Q}^{+}$and $\mathrm{Q}^{-}$separately. It will therefore suffice to prove that $\operatorname{sign}\left(\mathrm{Q}_{1}\right)$ and $\operatorname{sign}\left(\mathrm{Q}_{2}\right) \operatorname{lift} \operatorname{sign}(\mathrm{Q})$.

It is shown on [9, Lemma 1.2] that on the orthogonal complement of its kernel, the operator $\mathrm{Q}$ has compact resolvent. It follows that $\operatorname{sign}(\mathrm{Q})$ is a compact perturbation of the operator

$$
\mathrm{F}=\mathrm{Q}\left(\mathrm{I}+\mathrm{Q}^{2}\right)^{-\frac{1}{2}},
$$


and of course the same for $\mathrm{Q}_{1}$ and $\mathrm{Q}_{2}$. Now it is shown in [9, Proposition 1.1] that the operator $F$ commutes with the action of $\mathrm{C}(\mathrm{W})$ on $\mathrm{H}$, modulo compact operators, and the same argument shows that the operator

$$
\mathrm{F}_{1}=\mathrm{Q}_{1}\left(\mathrm{I}+\mathrm{Q}_{1}^{2}\right)^{-\frac{1}{2}}
$$

is a lift of F. Since they are compact perturbations, it follows that $\operatorname{sign}\left(Q_{1}\right)$ is a lift of $\operatorname{sign}(\mathrm{Q})$, and of course the same for $\mathrm{Q}_{2}$, as required.

Now let $\mathrm{P}, \mathrm{P}_{1}$ and $\mathrm{P}_{2}$ be the orthogonal projections onto the kernels of the operators $\mathrm{Q}^{+}, \mathrm{Q}_{1}^{+}$and $\mathrm{Q}_{2}^{+}$. These are the initial projections of the partial isometries $V, V_{1}$ and $V_{2}$. It follows from Lemma 8.20 that $P_{1}$ and $P_{2}$ are lifts of $P$, so that $\left(\mathrm{P}_{1}, \mathrm{P}_{2}\right) \in \mathrm{D}_{\mathrm{H}_{1}, \mathrm{H}_{2}}^{*}(\mathrm{~W})$.

8.21. Lemma. The projection $\left(\mathrm{P}_{1}, \mathrm{P}_{2}\right) \in \mathrm{D}_{\mathrm{H}_{1}, \mathrm{H}_{2}}^{*}(\mathrm{~W})$ defines the same class in the group $\mathcal{S}_{1}\left(\sigma_{1}, \sigma_{2}\right)$ as the analytic structure class of the cycle $(M, S, f, D, n)$.

Proof. For the most part we shall follow the related argument presented by Baum, Douglas and Taylor in [9, Proof of Proposition 4.3]. But we shall in addition need to keep track of Fredholm indices in a way that they did not, and to do this we shall use some computations from the monograph [11] of Booss-Bavnbek and Wojciechowski on elliptic boundary value theory.

Recall that, by definition, the range of $\mathrm{P}$ is the kernel of $\mathrm{Q}^{+}$. There is a continuous restriction-to-the-boundary, or trace map

$$
\tau: \text { Range }(\mathrm{P}) \longrightarrow \mathrm{H}^{-\frac{1}{2}}(\mathrm{M}, \mathrm{S})
$$

into the order $-\frac{1}{2}$ Sobolev space on $M=\partial W$ (see for example [11, Chapter 13]). We want to consider its composition with the pseudodifferential operator

$$
\mathrm{p}(\mathrm{D}): \mathrm{H}^{-\frac{1}{2}}(\mathrm{M}, \mathrm{S}) \longrightarrow \mathrm{H}^{-\frac{1}{2}}(\mathrm{M}, \mathrm{S}) .
$$

In fact, setting $R=\left(D^{2}+I\right)^{-\frac{1}{4}}$, we want to consider the commuting diagram

$$
\begin{aligned}
& \mathrm{L}^{2}\left(\mathrm{~W}, \mathrm{~S}^{+}\right) \stackrel{\mathrm{P}}{\longrightarrow} \operatorname{Range}(\mathrm{P}) \stackrel{\tau}{\longrightarrow} \mathrm{H}^{-\frac{1}{2}}(\mathrm{M}, \mathrm{S}) \stackrel{\mathrm{p}(\mathrm{D})}{\longrightarrow} \mathrm{H}^{-\frac{1}{2}}(\mathrm{M}, \mathrm{S}) \\
& R \downarrow \cong \\
& \mathrm{L}^{2}(\mathrm{M}, \mathrm{S}) \underset{\mathrm{p}(\mathrm{D})}{\longrightarrow} \mathrm{L}^{2}(\mathrm{M}, \mathrm{S}) \text {. }
\end{aligned}
$$

It is verified in the proof of [9, Proposition 4.3] that the composition determines a Fredholm operator from Range(P) to the range of $p(D)$ in $L^{2}(M, S)$. Moreover it 
is also verified there that the composition commutes, modulo compact operators, with the actions of $\mathrm{C}(\mathrm{W})$ on $\mathrm{L}^{2}\left(\mathrm{~W}, \mathrm{~S}^{+}\right)$and $\mathrm{L}^{2}(\mathrm{M}, \mathrm{S})$, and the same argument shows that the compositions associated to $\left(Q_{1}, D_{1}\right)$ and $\left(Q_{2}, D_{2}\right)$ are lifts of the composition for (Q,D).

We shall need to compute the Fredholm index of the operator

$$
\text { Range }(\mathrm{P}) \longrightarrow \text { Range }(\mathrm{p}(\mathrm{D}))
$$

determined by the composition. It follows from [11, Theorem 19.1] that the kernel of this map consists of smooth sections on $W$, and it is therefore the finite-dimensional space of those smooth sections in Range(P) that satisfy the APS boundary conditions described in Section 2. The cokernel may be identified with the APS cokernel by [11, Lemma 19.3 and Theorem 20.8]. It therefore follows that the index is equal to the APS index $\operatorname{Ind}(\mathrm{Q}, \mathrm{D})$.

Of course, the same conclusion can be reached for the pairs $\left(Q_{1}, D_{1}\right)$ and $\left(Q_{2}, D_{2}\right)$. If we let $e_{1}^{ \pm}$be any projections with ranks equal to the dimensions of the kernel and cokernel of the APS index problem for $\left(Q_{1}, D_{1}\right)$, and if we define $e_{2}^{ \pm}$similarly, then the operators just analyzed determine an equivalence of projections

$$
\left(P_{1}, P_{2}\right) \oplus\left(e_{1}^{-}, e_{2}^{-}\right) \sim\left(p\left(D_{1}\right), p\left(D_{2}\right)\right) \oplus\left(e_{1}^{+}, e_{2}^{+}\right)
$$

in the $\mathrm{C}^{*}$-algebra $\mathrm{D}_{\mathrm{H}_{1}, \mathrm{H}_{2}}^{*}(\mathrm{~W})$. We find that

$$
\left[\left(P_{1}, P_{2}\right)\right]=\left[\left(p\left(D_{1}\right), p\left(D_{2}\right)\right]+\left[\operatorname{Ind}\left(Q_{1}, D_{2}\right)\right]-\left[\operatorname{Ind}\left(Q_{2}, D_{2}\right)\right]\right.
$$

at the level of K-theory (recall Definition 8.6, which associates a K-theory class to each integer). The lemma therefore follows from the definition of the analytic structure class (8.17) and the definition of a boundary cycle (8.9).

The proof of Proposition 8.19 is now completed by the following two observations:

8.22. Lemma. The partial isometry $\mathrm{V}: \mathrm{L}^{2}\left(\mathrm{~W}, \mathrm{~S}^{+}\right) \rightarrow \mathrm{L}^{2}\left(\mathrm{~W}, \mathrm{~S}^{-}\right)$is surjective, as are the partial isometries $\mathrm{V}_{1}$ and $\mathrm{V}_{2}$.

Proof. We shall consider only $\mathrm{V}$ (the proofs for $\mathrm{V}_{1}$ and $\mathrm{V}_{2}$ are exactly the same). The range of $\mathrm{V}$ is the closure of range of $\mathrm{Q}^{+}$, and so we need to show that the orthogonal complement of the range of $\mathrm{Q}^{+}$is zero. But this is the kernel of the operator $\mathrm{Q}^{-}$(the odd-to-even component of $\mathrm{Q}$ ) considered as a Hilbert 
space operator with its minimal domain. A section $s$ lies in the kernel of $\mathrm{Q}^{-}$if and only if there is a sequence of smooth sections $s_{n}$ supported in the interior of $W$ such that $\left\|s_{n}-s\right\| \rightarrow 0$ and $\left\|Q^{-} s_{n}\right\| \rightarrow 0$. If we extend $W$ to a closed manifold $Z$ as in Section 2, and if we extend sections by zero from $W$ to $Z$, then the extended $s_{n}$ are smooth sections on $Z$, and we still have $\left\|s_{n}-s\right\| \rightarrow 0$ and $\left\|Q^{-} s_{n}\right\| \rightarrow 0$. By elliptic regularity, $s$ is a smooth section on $Z$. Since $s$ vanishes on the complement of $W$ in $Z$, the unique continuation property for solutions of Dirac equations implies that $s \equiv 0$ (see [11, Chapter 8]).

8.23. Lemma. The projection $\left(\mathrm{P}_{1}, \mathrm{P}_{2}\right) \in \mathrm{D}_{\mathrm{H}_{1}, \mathrm{H}_{2}}^{*}(\mathrm{~W})$ defines the zero class in the structure group $\mathcal{S}_{1}\left(\sigma_{1}, \sigma_{2}\right)$.

Proof. Observe that

$$
\mathrm{P}_{1}=\mathrm{I}_{1}^{+}-\mathrm{V}_{1}^{*} \mathrm{~V}_{1} \quad \text { and } \quad \mathrm{P}_{2}=\mathrm{I}_{2}^{+}-\mathrm{V}_{2}^{*} \mathrm{~V}_{2}
$$

where $\mathrm{I}_{1}^{+}$and $\mathrm{I}_{2}^{+}$denote the identity operators on the even-graded components of the Hilbert spaces $\mathrm{H}_{1}$ and $\mathrm{H}_{2}$. Using the obvious extension of this notation, Lemma 8.22 shows that

$$
\mathrm{V}_{1} \mathrm{~V}_{1}^{*}=\mathrm{I}_{1}^{-} \quad \text { and } \quad \mathrm{V}_{2} \mathrm{~V}_{2}^{*}=\mathrm{I}_{2}^{-} .
$$

So at the level of K-theory we find that

$$
\left[\left(\mathrm{P}_{1}, \mathrm{P}_{2}\right)\right]=\left[\left(\mathrm{I}_{1}^{+}, \mathrm{I}_{2}^{+}\right)\right]-\left[\left(\mathrm{I}_{1}^{-}, \mathrm{I}_{2}^{-}\right)\right] \text {. }
$$

The classes on the right hand side are zero by Example 4.3.

The remaining parts of the proof of Theorem 8.18 are much simpler:

Proof of Theorem 8.18. It is straightforward to show that cycles that are equivalent via direct sum/disjoint union have the same analytic structure class, and we have dealt with bordism in Proposition 8.19. It therefore remains to show that if $(\hat{M}, \hat{S}, \hat{f}, \widehat{D}, n)$ and $(M, S, f, D, n)$ are equivalent via bundle modification, as in item (c) of Definition 8.13, then their analytic structure classes are equal. Recall from the proof of Proposition 6.6 that we may decompose $\hat{\mathrm{D}}_{1}$ and $\widehat{\mathrm{D}}_{2}$ as orthogonal direct sums

$$
\widehat{\mathrm{D}}_{1}=\mathrm{P} \hat{\mathrm{D}}_{1} \mathrm{P}+\mathrm{P}^{\perp} \hat{\mathrm{D}}_{1} \mathrm{P}^{\perp} \text { and } \hat{\mathrm{D}}_{2}=\mathrm{P}_{2} \mathrm{P}+\mathrm{P}^{\perp} \hat{\mathrm{D}}_{2} \mathrm{P}^{\perp}
$$

The first summands identify with $\mathrm{D}_{1}$ and $\mathrm{D}_{2}$, and it suffices to show that the second summands determine the zero element in K-theory. 
Let us use the abbreviated notation $E_{1}$ and $E_{2}$ for $\mathrm{P}^{\perp} \hat{\mathrm{D}}_{1} \mathrm{P}^{\perp}$ and $\mathrm{P}^{\perp} \hat{\mathrm{D}}_{1} \mathrm{P}^{\perp}$. These are invertible self-adjoint operators, and so

$$
p\left(E_{1}\right)=\frac{1}{2}\left(\operatorname{sign}\left(E_{1}\right)+I\right) \text { and } p\left(E_{1}\right)=\frac{1}{2}\left(\operatorname{sign}\left(E_{2}\right)+I\right) .
$$

We must show that if we set $H=P^{\perp} L^{2}(\widehat{M}, \widehat{S})$, then the projection

$$
\left(p\left(E_{1}\right), p\left(E_{2}\right)\right) \in D_{H_{1}, H_{2}}^{*}(X)
$$

determines the zero K-theory class. The self-adjoint involutions $\operatorname{sign}\left(E_{1}\right)$ and $\operatorname{sign}\left(E_{1}\right)$ anticommute with the self-adjoint involutions $J_{1}$ and $J_{2}$ introduced in the proof of Proposition 6.6, and we can therefore form the path of self-adjoint involutions

$$
\left(s \operatorname{sign}\left(E_{1}\right)+t J_{1}, s \operatorname{sign}\left(E_{1}\right)+t J_{2}\right) \quad\left(s, t \geq 0, \quad s^{2}+t^{2}=1\right)
$$

in $\mathrm{D}_{\mathrm{H}_{1}, \mathrm{H}_{2}}^{*}(\mathrm{X})$. Looking at the corresponding path of positive spectral projections, we find that $\left(p\left(E_{1}\right), p\left(E_{2}\right)\right)$ is path-connected to

$$
\left(\mathrm{Q}_{1}, \mathrm{Q}_{2}\right)=\left(\frac{1}{2}\left(\mathrm{~J}_{1}+\mathrm{I}\right), \frac{1}{2}\left(\mathrm{~J}_{2}+\mathrm{I}\right)\right) .
$$

But this projection is degenerate in the sense of Example 4.3 and so is zero in K-theory.

We therefore obtain a map $\mathcal{S}_{1}^{\text {geom }}\left(\sigma_{1}, \sigma_{2}\right) \rightarrow \mathcal{S}_{1}\left(\sigma_{1}, \sigma_{2}\right)$ by associating to any geometric cycle its analytic structure class. It remains only to show that this map is an isomorphism.

8.24. Lemma. There is an exact sequence

$$
\mathbb{Z} \longrightarrow \mathcal{S}_{1}^{\text {geom }}\left(\sigma_{1}, \sigma_{2}\right) \longrightarrow \mathrm{K}_{1}(\mathrm{~B} \pi) \longrightarrow 0
$$

in which $\mathrm{n} \in \mathbb{Z}$ is mapped to the class of the unique geometric cycle $(M, S, f, D, n)$ with $M=\emptyset$, and the class of any cycle $(M, S, f, D, n)$ maps to the class of $(M, S, f)$.

Proof. Because of the bordism relation, if $(M, S, f)$ is any geometric K-cycle for $B \pi$, if $D$ and $D^{\prime}$ are Dirac operators for this cycle, and if $n \in \mathbb{Z}$, then there is some $n^{\prime} \in \mathbb{Z}$ such that $(M, S, f, D, n)$ and $\left(M, S, f, D^{\prime}, n^{\prime}\right)$ are equivalent. As a result, if $(M, S, f)$ is equivalent to $\left(M^{\prime}, S^{\prime}, f^{\prime}\right)$, then any geometric $\left(\sigma_{1}, \sigma_{2}\right)$-cycle $(M, S, f, D, n)$ is equivalent to some cycle of the form $\left(M^{\prime}, S^{\prime}, f^{\prime}, D^{\prime}, n^{\prime}\right)$. The lemma follows from this. 
8.25. Proposition. The group homomorphism

$$
\mathcal{S}_{1}^{\text {geom }}\left(\sigma_{1}, \sigma_{2}\right) \rightarrow \mathcal{S}_{1}\left(\sigma_{1}, \sigma_{2}\right)
$$

that associates to any geometric $\left(\sigma_{1}, \sigma_{2}\right)$-cycle its analytic structure class is an isomorphism.

Proof. This follows from the commuting diagram

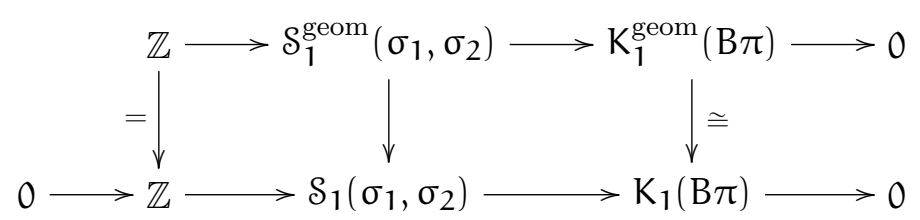

and the five lemma.

\section{Proofs of the Main Theorems}

We have all the pieces in place to carry out the program sketched in Section 1.

Proof of Theorem 1.1. Let $M$ be an odd-dimensional, closed Riemannian spin manifold with positive scalar curvature. As outlined in the introduction, it suffices to show that if $D$ is the Dirac operator on $M$, then the relative trace of the structure invariant $[M, f] \in \mathcal{S}_{1}(\pi)$ of Definition 7.1 is equal to the relative eta invariant $\rho\left(D, f, \sigma_{1}, \sigma_{2}\right)$. Let us examine the diagram

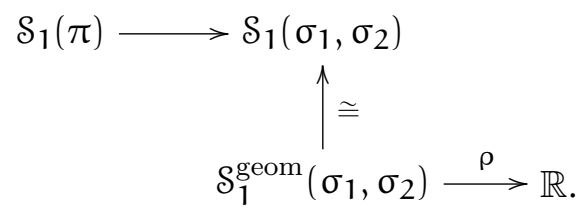

that is used to define the relative trace. The class $[M, f]$ maps to the class $\left[\left(p\left(D_{1}\right), p\left(D_{2}\right)\right]\right.$ in $\mathcal{S}_{1}\left(\sigma_{1}, \sigma_{2}\right)$, and this is by definition the image of the geometric structure class $(M, S, f, D, 0)$. Applying $\rho$ we obtain, by definition, the relative eta invariant $\rho\left(D, f, \sigma_{1}, \sigma_{2}\right)$.

Proof of Theorem 1.2. The argument is essentially the same as the one just given, although a little more involved. We shall analyze what becomes of the structure invariant $\left[h, f^{\prime}, f^{\prime \prime}\right] \in \mathcal{S}_{1}(\pi)$ as we apply the various steps that make up the definition of the relative trace map, and thereby compute that the relative trace of $\left[h, f^{\prime}, f^{\prime \prime}\right]$ is the difference of relative eta invariants. 
The invariant $\left[h, f^{\prime}, f^{\prime \prime}\right]$ is given by the pair $\left(\tilde{P}_{\pi}, \gamma_{\pi}\right)$, where $\tilde{P}_{\pi}$ is the operator of Example 7.5 that is associated with the direct sum

$$
\mathrm{D}=\mathrm{D}^{\prime} \oplus-\mathrm{D}^{\prime \prime}
$$

of signature operators on $M^{\prime}$ and $M^{\prime \prime}$, and $\gamma_{\pi}$ is the path described in Section 7 that connects the Cayley transform of $\mathrm{D}_{\pi}$ to the identity.

The image of $\left[h, f^{\prime}, f^{\prime \prime}\right]$ in $\mathcal{S}_{1}\left(\sigma_{1}, \sigma_{2}\right)$ may be described, using Lemma 7.4 , by the element $\left(\tilde{\mathrm{P}}_{1}, \tilde{\mathrm{P}}_{2}\right) \in \mathrm{D}_{\mathrm{H}_{1}, \mathrm{H}_{2}}^{*}(\mathrm{X})$ together with the path $\left(\gamma_{1}, \gamma_{2}\right)$ obtained from $\gamma_{\pi}$ that connects the Cayley transforms of $D_{1}$ and $D_{2}$ to the identity operators on $\mathrm{H}_{1}$ and $\mathrm{H}_{2}$.

We are going to identify this class in $\delta_{1}\left(\sigma_{1}, \sigma_{2}\right)$ with the analytic structure class of the geometric $\left(\sigma_{1}, \sigma_{2}\right)$-cycle

$$
\left(M^{\prime}, S^{\prime}, f^{\prime}, D^{\prime}, 0\right) \sqcup-\left(M^{\prime \prime}, S^{\prime \prime}, f^{\prime \prime}, D^{\prime \prime}, 0\right) .
$$

This will complete the proof since

$$
\begin{aligned}
\tau:\left(M^{\prime}, S^{\prime}, f^{\prime}, D^{\prime}, 0\right) \sqcup-\left(M^{\prime \prime}, S^{\prime \prime}, f^{\prime \prime}, D^{\prime \prime}, 0\right) & \mapsto \\
& \rho\left(D^{\prime}, f^{\prime}, \sigma_{1}, \sigma_{2}\right)-\rho\left(D^{\prime \prime}, f^{\prime \prime}, \sigma_{1}, \sigma_{2}\right) .
\end{aligned}
$$

In the calculation that follows we shall streamline notation by referring to $D$, rather than $\left(D_{1}, D_{2}\right)$, and $\gamma$ rather than $\left(\gamma_{1}, \gamma_{2}\right)$.

In the definition of the path $\gamma_{\pi}$ we used the heat kernel to obtain from the homotopy equivalence $h$ a map

$$
A_{\pi}=\exp \left(-\Delta_{\pi}^{\prime \prime}\right) h^{*} \exp \left(-\Delta_{\pi}^{\prime}\right): \mathrm{L}_{\pi}^{2}\left(M^{\prime}, S^{\prime}\right) \longrightarrow \mathrm{L}_{\pi}^{2}\left(M^{\prime \prime}, S^{\prime \prime}\right) .
$$

Having passed from Hilbert modules to Hilbert spaces to go from $\mathcal{S}_{1}(\pi)$ to $\mathcal{S}_{1}\left(\sigma_{1}, \sigma_{2}\right)$, we may replace these heat kernels with the projections $R$ onto the subspaces of harmonic forms. Thus we may redefine

$$
A:=R^{\prime \prime} h^{*} R^{\prime}: L^{2}\left(M^{\prime}, S^{\prime}\right) \longrightarrow L^{2}\left(M^{\prime \prime}, S^{\prime \prime}\right) .
$$

The straight line path from $\exp (-\Delta)$ to $R$ determines a fixed end-point homotopy from the old path $\gamma$ to the new one. Having made this change, the operator D and the path $\gamma$ decompose as direct sums with respect to the decomposition of the underlying Hilbert space $\mathrm{H}$ into the harmonic forms, direct sum the orthogonal complement of the harmonic forms. 
On the orthogonal complement of the harmonic forms, the path $\gamma$ is given by the formula $\left(\mathbb{B}-\mathbb{J}_{t}\right)\left(\mathbb{B}+\mathbb{J}_{t}\right)^{-1}$ on $[0,1]$, where

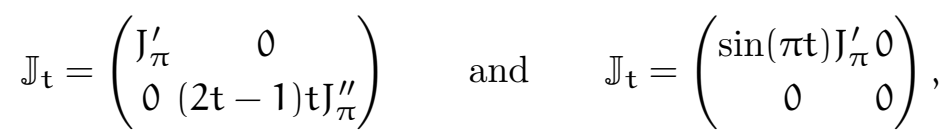

on $\left[0, \frac{1}{2}\right]$ and $\left[\frac{1}{2}, 1\right]$, respectively (in addition, the path $\gamma$ is constant on $[1,2]$ ). Thus $\gamma$ is fixed-endpoint homotopic to the simpler path $(\mathbb{B}-s \mathbb{J})(\mathbb{B}+s \mathbb{J})^{-1}$, where $s=\left(1-\frac{1}{2} t\right)$ and $t \in[0,2]$. Since

$$
(\mathbb{B}-s \mathbb{J})(\mathbb{B}+s \mathbb{J})^{-1}=\left(s^{-1} \mathrm{D}-\mathrm{iI}\right)\left(\mathrm{s}^{-1} \mathrm{D}+\mathrm{iI}\right)^{-1},
$$

and since the function $\frac{2}{\pi} \arctan \left(s^{-1} x\right)$ converges uniformly to sign $(x)$ on the complement of any neighborhood of $0 \in \mathbb{R}$ as $s \rightarrow 0$, we find that on the orthogonal complement of the harmonic forms, the structure determined $\tilde{P}$ and the path $\gamma$ is equal to the one determined by the projection $p(D)$ and the constant path I.

On the finite-dimensional space of harmonic forms the operator $\tilde{P}$ is equal to $\frac{1}{2} \mathrm{I}$, so that $\exp (2 \pi i \tilde{P})=-\mathrm{I}$. Since $\mathbb{B}=0$ on harmonic forms, the path $\gamma$, which is given by

$$
\left(\mathbb{B}-\mathbb{J}_{\mathfrak{t}}\right)\left(\mathbb{B}+\mathbb{J}_{\mathfrak{t}}\right)^{-1} \quad \text { and } \quad\left(\mathbb{B}-\mathbb{J}_{1}\right)\left(\mathbb{B}+\mathbb{J}_{t}\right)^{-1}
$$

on the intervals $[0,1]$ and $[1,2]$, respectively, is simply $-\mathbb{J}_{t} \mathbb{J}_{t}^{-1}$ and $-\mathbb{J}_{1} \mathbb{J}_{t}^{-1}$ on these intervals. The first expression gives the constant path $-\mathrm{I}$, while a quick calculation shows that the second gives

$$
\left(\begin{array}{cc}
e^{-\pi i t} I & 0 \\
0 & e^{\pi i t} I
\end{array}\right) \quad(t \in[1,2]) .
$$

Now as $s$ ranges through $[0,1]$, deform $\tilde{P}=\frac{1}{2} \mathrm{I}$ and $\gamma$ as above through

$$
\left(\begin{array}{cc}
\frac{1-s}{2} \mathrm{I} & 0 \\
0 & \frac{1+s}{2} \mathrm{I}
\end{array}\right) \text { and }\left(\begin{array}{cc}
e^{-\pi i(t-s t+2 s)} \mathrm{I} & 0 \\
0 & e^{\pi i(t-s t+2 s)} \mathrm{I}
\end{array}\right)
$$

(we have given the formula for deformed paths in the range $t \in[1,2]$; for $t \in[0,1]$ the paths are constant, for each $s$ ). Setting $s=1$, we find that the structure given by the element $\tilde{P}$ and path $\gamma$ is equal to the structure given by the projection $\left(\begin{array}{ll}0 & 0 \\ 0 & \mathrm{I}\end{array}\right)$ and the constant path.

Putting the two orthogonal summands together, we find that the structure class in $\mathcal{S}_{1}\left(\sigma_{1}, \sigma_{2}\right)$ determined by $\tilde{\mathrm{P}}_{\pi}$ and $\gamma_{\pi}$ is equal to the class determined by 
the projection

$$
P=\left(\begin{array}{cc}
p\left(D^{\prime}\right) & 0 \\
0 & p\left(-D^{\prime \prime}\right)+p^{\prime \prime}
\end{array}\right),
$$

and the constant path, where $\mathrm{p}^{\prime \prime}$ is the projection onto the kernel of $\mathrm{D}^{\prime \prime}$. But bearing in mind that

$$
-\left(M^{\prime \prime}, S^{\prime \prime}, f^{\prime \prime}, D^{\prime \prime}, 0\right)=\left(M^{\prime \prime},-S^{\prime \prime}, f^{\prime \prime},-D^{\prime \prime}, d_{1}-d_{2}\right),
$$

we find that this is precisely the analytic structure class of the geometric cycle

$$
\left(M^{\prime}, S^{\prime}, f^{\prime}, D^{\prime}, 0\right) \sqcup-\left(M^{\prime \prime}, S^{\prime \prime}, f^{\prime \prime}, D^{\prime \prime}, 0\right) .
$$

as required.

\section{REFERENCES}

[1] M. F. Atiyah. Global theory of elliptic operators. In Proc. Internat. Conf. on Functional Analysis and Related Topics (Tokyo, 1969), pages 21-30. Univ. of Tokyo Press, Tokyo, 1970.

[2] M. F. Atiyah, V. K. Patodi, and I. M. Singer. Spectral asymmetry and Riemannian geometry. Bull. London Math. Soc., 5:229-234, 1973.

[3] M. F. Atiyah, V. K. Patodi, and I. M. Singer. Spectral asymmetry and Riemannian geometry. I. Math. Proc. Cambridge Philos. Soc., 77:43-69, 1975.

[4] M. F. Atiyah, V. K. Patodi, and I. M. Singer. Spectral asymmetry and Riemannian geometry. II. Math. Proc. Cambridge Philos. Soc., 78(3):405-432, 1975.

[5] M. F. Atiyah, V. K. Patodi, and I. M. Singer. Spectral asymmetry and Riemannian geometry. III. Math. Proc. Cambridge Philos. Soc., 79(1):71-99, 1976.

[6] P. Baum and A. Connes. Geometric K-theory for Lie groups and foliations. Enseign. Math. (2), 46(1-2):3-42, 2000.

[7] P. Baum, A. Connes, and N. Higson. Classifying space for proper actions and K-theory of group C*-algebras. In C*-algebras: 1943-1993 (San Antonio, TX, 1993), volume 167 of Contemp. Math., pages 240-291. Amer. Math. Soc., Providence, RI, 1994.

[8] P. Baum and R. G. Douglas. K homology and index theory. In Operator algebras and applications, Part I (Kingston, Ont., 1980), volume 38 of Proc. Sympos. Pure Math., pages 117-173. Amer. Math. Soc., Providence, R.I., 1982.

[9] P. Baum, R. G. Douglas, and M. E. Taylor. Cycles and relative cycles in analytic Khomology. J. Differential Geom., 30(3):761-804, 1989.

[10] P. Baum, N. Higson, and T. Schick. On the equivalence of geometric and analytic Khomology. Pure Appl. Math. Q., 3(1):1-24, 2007.

[11] B. Booß-Bavnbek and K. P. Wojciechowski. Elliptic boundary problems for Dirac operators. Mathematics: Theory \& Applications. Birkhäuser Boston Inc., Boston, MA, 1993.

[12] L. G. Brown, R. G. Douglas, and P. A. Fillmore. Extensions of C*-algebras and K-homology. Ann. of Math. (2), 105(2):265-324, 1977. 
[13] P.-A. Cherix, M. Cowling, P. Jolissaint, P. Julg, and A. Valette. Groups with the Haagerup property, volume 197 of Progress in Mathematics. Birkhäuser Verlag, Basel, 2001.

[14] A. Connes and G. Skandalis. The longitudinal index theorem for foliations. Publ. Res. Inst. Math. Sci., 20(6):1139-1183, 1984.

[15] X. Dai and W. Zhang. Higher spectral flow. J. Funct. Anal., 157(2):432-469, 1998.

[16] M. S. Farber and J. P. Levine. Jumps of the eta-invariant. Math. Z., 223(2):197-246, 1996. With an appendix by Shmuel Weinberger: Rationality of $\rho$-invariants.

[17] P. B. Gilkey. Invariance theory, the heat equation, and the Atiyah-Singer index theorem. Studies in Advanced Mathematics. CRC Press, Boca Raton, FL, second edition, 1995.

[18] E. Guentner, N. Higson, and S. Weinberger. The Novikov conjecture for linear groups. Publ. Math. Inst. Hautes Études Sci., (101):243-268, 2005.

[19] N. Higson. C*-algebra extension theory and duality. J. Funct. Anal., 129(2):349-363, 1995.

[20] N. Higson and G. Kasparov. E-theory and KK-theory for groups which act properly and isometrically on Hilbert space. Invent. Math., 144(1):23-74, 2001.

[21] N. Higson and J. Roe. Analytic K-homology. Oxford Mathematical Monographs. Oxford University Press, Oxford, 2000.

[22] N. Higson and J. Roe. Mapping surgery to analysis. I. Analytic signatures. K-Theory, 33(4):277-299, 2005.

[23] N. Higson and J. Roe. Mapping surgery to analysis. II. Geometric signatures. K-Theory, 33(4):301-324, 2005.

[24] N. Higson and J. Roe. Mapping surgery to analysis. III. Exact sequences. K-Theory, 33(4):325-346, 2005.

[25] G. G. Kasparov. Topological invariants of elliptic operators. I. K-homology. Izv. Akad. Nauk SSSR Ser. Mat., 39(4):796-838, 1975.

[26] N. Keswani. Geometric K-homology and controlled paths. New York J. Math., 5:53-81 (electronic), 1999.

[27] N. Keswani. Relative eta-invariants and C*-algebra K-theory. Topology, 39(5):957-983, 2000.

[28] E. C. Lance. Hilbert C*-modules, volume 210 of London Mathematical Society Lecture Note Series. Cambridge University Press, Cambridge, 1995.

[29] V. Mathai. Spectral flow, eta invariants, and von Neumann algebras. J. Funct. Anal., 109(2):442-456, 1992.

[30] A. S. Miščenko. Infinite-dimensional representations of discrete groups, and higher signatures. Izv. Akad. Nauk SSSR Ser. Mat., 38:81-106, 1974.

[31] W. L. Paschke. K-theory for commutants in the Calkin algebra. Pacific J. Math., 95(2):427434, 1981.

[32] P. Piazza and T. Schick. Bordism, rho-invariants and the Baum-Connes conjecture. J. Noncommut. Geom., 1(1):27-111, 2007.

[33] A. Ranicki. Algebraic and geometric surgery. Oxford Mathematical Monographs. Oxford University Press, Oxford, 2002.

[34] J. Roe. Notes on regularity for Dirac operators on bundles of Hilbert C*-modules. Unpublished. Available at http://www.math.psu.edu/roe.

[35] G. Skandalis. Some remarks on Kasparov theory. J. Funct. Anal., 56(3):337-347, 1984. 
[36] C. T. C. Wall. Surgery on compact manifolds, volume 69 of Mathematical Surveys and Monographs. American Mathematical Society, Providence, RI, second edition, 1999. Edited and with a foreword by A. A. Ranicki.

[37] S. Weinberger. Homotopy invariance of $\eta$-invariants. Proc. Nat. Acad. Sci. U.S.A., 85(15):5362-5363, 1988.

Nigel Higson and John Roe

Department of Mathematics

Penn State University

University Park

PA 16802

E-mail: higson@math.psu.edu 